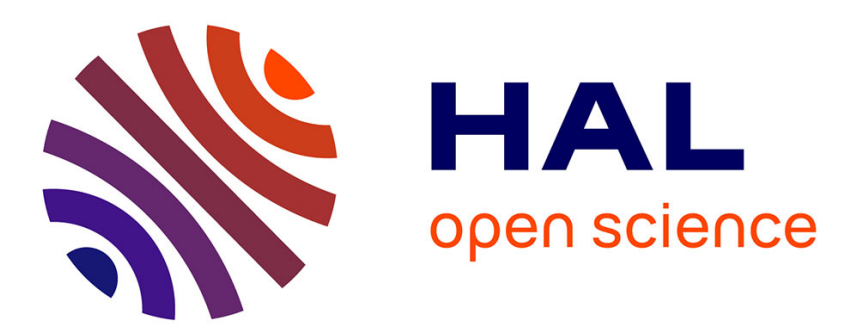

\title{
Coexistence phenomena and global bifurcation structure in a chemostat-like model with species-dependent diffusion rates \\ François Castella, Sten Madec
}

\section{- To cite this version:}

François Castella, Sten Madec. Coexistence phenomena and global bifurcation structure in a chemostat-like model with species-dependent diffusion rates. Journal of Mathematical Biology, 2014, 68 (1-2), pp.377-415. 10.1007/s00285-012-0633-7 . hal-00777025

\section{HAL Id: hal-00777025 \\ https://hal.science/hal-00777025}

Submitted on 17 Jan 2013

HAL is a multi-disciplinary open access archive for the deposit and dissemination of scientific research documents, whether they are published or not. The documents may come from teaching and research institutions in France or abroad, or from public or private research centers.
L'archive ouverte pluridisciplinaire HAL, est destinée au dépôt et à la diffusion de documents scientifiques de niveau recherche, publiés ou non, émanant des établissements d'enseignement et de recherche français ou étrangers, des laboratoires publics ou privés. 


\title{
Coexistence phenomena and global bifurcation structure in a chemostat-like model with species-dependent diffusion rates
}

\author{
François Castella • Sten Madec
}

the date of receipt and acceptance should be inserted later

\begin{abstract}
We study the competition of two species for a single resource in a chemostat. In the simplest space-homogeneous situation, it is known that only one species survives, namely the best competitor. In order to exhibit coexistence phenomena, where the two competitors are able to survive, we consider a space dependent situation: we assume that the two species and the resource follow a diffusion process in space, on top of the competition process. Besides, and in order to consider the most general case, we assume each population is associated with a distinct diffusion constant. This is a key difficulty in our analysis: the specific (and classical) case where all diffusion constants are equal, leads to a particular conservation law, which in turn allows to eliminate the resource in the equations, a fact that considerably simplifies the analysis and the qualitative phenomena.

Using the global bifurcation theory, we prove that the underlying 2-species, stationary, diffusive, chemostat-like model, does possess coexistence solutions, where both species survive. On top of that, we identify the domain, in the space of the identified bifurcation parameters, for which the system does have coexistence solutions.
\end{abstract}

Keywords Global bifurcation · Elliptic systems · Heterogeneous environment · Coexistence - Chemostat

Mathematics Subject Classification (2000) 35Q92 - 35K58 - 92D25 - 92D30

\section{Introduction}

The present paper is devoted to the study of coexistence solutions in some chemostatlike systems, where various species compete for a single resource. The starting point

Sten Madec

Institut de Mathematiques de Bordeaux Universite Victor Segalen Bordeaux 2, 3ter place de la victoire, 33000 Bordeaux Cedex, France

E-mail: sten.madec@univ-rennes1.fr

François Castella

Université de Rennes 1, UMR CNRS 6625 Irmar, Campus de Beaulieu, 35042 Rennes cedex, France

E-mail: francois.castella@univ-rennes1.fr 
of our analysis is the fact that in the simplest models, i.e. in the space-homogeneous situation, only one species survives, namely the best competitor. Therefore, and in order to observe situations where all species are able to survive, we readily consider the space-inhomogeneous situation, where the various species and the single resource follow a diffusion process in space. Technically speaking, and in order to tackle the most general situation, we assume that each population possesses its own distinct diffusion coefficient. This is a major difficulty and originality in the present text, as we discuss later in this introduction.

The main result of this paper is that the underlying 2-species chemostat-like model, does possess coexistence solutions, i.e. solutions where all species survive. Besides, we are able to identify a domain in the space of the relevant parameters, for which coexistence holds.

Our construction relies on global bifurcations in elliptic systems. Although we conjecture that our analysis may be generalized to the case of $N$ competing species for any $N \geq 2$, our results can only be proved in the case $N=2$ for the time being.

Let us come to technical statements.

We study the nonnegative steady-state solutions of the reaction-diffusion system

$$
\left\{\begin{array}{l}
\partial_{t} R=a_{0} \Delta R-F_{1}(x, R) U-F_{2}(x, R) V-m_{0}(x) R+I, \\
\partial_{t} U=a_{1} \Delta U+\left(F_{1}(x, R)-m_{1}(x)\right) U, \\
\partial_{t} V=a_{2} \Delta V+\left(F_{2}(x, R)-m_{2}(x)\right) V,
\end{array} \quad(x \in \Omega, \quad t>0),\right.
$$

where $\Omega$ is a bounded region in $\mathbb{R}^{n}$ with smooth boundary. The above system is supplemented with Neumann ${ }^{1}$ boundary conditions

$$
\partial_{n} R(t, x)=\partial_{n} U(t, x)=\partial_{n} V(t, x)=0 \quad(x \in \partial \Omega, \quad t>0),
$$

where $\partial_{n}$ is the normal derivative on the boundary $\partial \Omega$.

The above system describes a situation where two species with density $U=U(t, x)$ and $V=V(t, x)$ respectively, compete for the same resource with density $R=R(t, x)$, through the nonlinear terms $F_{i}(x, R) U$ and $F_{i}(x, R) V(i=1,2)$. Besides, the space dependent resource $R$, as well as the two species $U, V$, follow a diffusion process in space, with the distinct diffusion constants $a_{0}>0, a_{1}>0, a_{2}>0$ respectively $\left.\right|^{2}$ The space dependent functions $m_{i}(x)>0$ on $\bar{\Omega}(i=0,1,2)$, are death rates, while the space dependent functions $F_{i}(x, R)=F_{i}(x, R(t, x)) \geq 0$ are the consumption rates. The given, time-independent function $I=I(x) \geq 0$ is the nutrient input. All these data are assumed smooth.

In order to implement a bifurcation method, we normalize the consumption rates as follows. We readily choose given, smooth, functions $f_{1}=f_{1}(x, R), f_{2}=f_{2}(x, R)$, and introduce two bifurcation parameters $c_{1}>0$ and $c_{2}>0$, which somehow measure the strength of the interaction between the species and the resource, through

$$
F_{1}(x, R) \equiv c_{1} f_{1}(x, R), \quad F_{2}(x, R) \equiv c_{2} f_{2}(x, R) .
$$

1 Robin boundary conditions, of the form $a_{0} \partial_{n} R+b_{0}(x) R=g(x), a_{1} \partial_{n} U+b_{1}(x) U=$ $a_{2} \partial_{n} V+b_{1}(x) V=0$ on $\partial \Omega$, with $g(x) \geq 0$ and $b_{i}(x) \geq 0(i=0,1,2)$, would do as well, as we discuss later in this text.

2 Our analysis is valid when the various constant coefficients diffusion operators $a_{i} \Delta$ become $\operatorname{div} a_{i}(x) \nabla$ for some smooth, space-dependent coefficients $a_{i}(x)>0$ on $\bar{\Omega}$, provided all coefficients $a_{i}(x)$ are proportional, i.e. $a_{i}(x)=\lambda_{i} a_{0}(x)(i=1,2)$ for some constants $\lambda_{1}>0$ and $\lambda_{2}>0$. This easy extension is discussed later in the text. Needless to say, in that case, Robin boundary conditions become $a_{0}(x) \partial_{n} R+b_{0}(x) R=g(x)$ on $\partial \Omega$, and so on, with $g(x) \geq 0$ and $b_{i}(x) \geq 0$ on $\partial \Omega(i=0,1,2)$. 
Note that, since we are only interested in nonnegative solutions $(R, U, V)$, the only important data is the value of $f_{i}(x, R)$ for $R \geq 0$ : as shown by our analysis, any smooth extension of $f_{i}(x, R)$ may be retained for values $R \leq 0$, provided $f_{i}(x, R) \leq 0$ whenever $R \leq 0$.

With the above notations, in this paper we look for stationary solutions $U=U(x)$, $V=V(x), R=R(x)$ to the above system, namely ${ }^{3}$

$$
\begin{cases}\left(m_{0}(x)-a_{0} \Delta\right) R+c_{1} f_{1}(x, R) U+c_{2} f_{2}(x, R) V=I(x), & \\ \left(m_{1}(x)-a_{1} \Delta\right) U-c_{1} f_{1}(x, R) U=0, & (x \in \Omega) \\ \left(m_{2}(x)-a_{2} \Delta\right) V-c_{2} f_{2}(x, R) V=0, & (x \in \partial \Omega) . \\ \partial_{n} R=\partial_{n} U=\partial_{n} V=0 & \end{cases}
$$

More precisely, our goal is to exhibit coexistence solutions in 1.2 , i.e. solutions $R, U$, $V$ for which $R>0, U>0, V>0$. Our approach relies on a global bifurcation method, where $c_{1}$ and $c_{2}$ are used as bifurcation parameters. In that respect, we also aim at identifying a domain in the $\left(c_{1}, c_{2}\right)$-plane for which coexistence holds.

Let us come to some bibliographical comments.

Bifurcation methods have been used in many texts concerning interacting species (competition models, predator-prey systems), see [20,21,23,22] and more recently in the study of some age structured models, see [8, 9]. In that respect, we wish to stress that the chemostat involves a fairly specific mathematical structure, a fact that plays a crucial role below: the nonlinear coupling in 1.2 , say, only involves terms of the form $f_{i}(x, R) U$ or $f_{i}(x, R) V$; in other words the two species $U$ and $V$ in $(1.2)$ are only coupled through the resource $R$. This observation holds in any chemostat model and allows, in some situations, to reduce the original model to a standard competition system by eliminating the equation on the resource, see [13, 12, 11, $, 18,19,10$.

Steady states of unstirred chemostats have been first studied by Waltman et al. in 11. The authors consider two species evolving in the one-dimensional situation $\Omega=[0,1]$. A generalisation in the case of two species evolving in a higher dimensional domain $\Omega$ is studied by $\mathrm{Wu}[18$ and $\mathrm{Wu}$ and Nie [19]. Using the index in a positive cone (see [24), Zheng et al. 15, 14 show coexistence results in systems with various trophic levels. In all these texts, the heterogeneity in space, that is crucial to recover coexistence phenomena, is introduced by imposing a gradient of the resource, which in turn is obtained through the boundary condition, of Robin type. All other coefficients are space independent. In the present text at variance, we allow the reaction terms (and other less crucial coefficients) to actually depend on space.

A key point is the following. In all the above works, the authors assume that the competing species, and the resource, have the same diffusion rate and the same death rate. This assumption provides a specific conservation law, that links the resource and the competing species. In our case it reads (taking $a_{0}=a_{1}=a_{2}=a$ and $m_{0}(x)=$ $\left.m_{1}(x)=m_{2}(x)=m(x)\right)$

$$
m(x)(R+U+V)-a \Delta(R+U+V)=I(x) .
$$

Relation 1.3 allows to eliminate the resource $R$ from the equations, and to write a reduced system whose semi-trivial solutions satisfy a simple, scalar, elliptic equation.

\footnotetext{
3 Recall that Robin boundary conditions are covered by our analysis, as well as variable coefficients diffusion operators div $a_{i}(x) \nabla$, provided $a_{i}(x)=\lambda_{i} a_{0}(x)(i=1,2)$, see footnotes 1 and 2
} 
Semi-trivial solutions are those corresponding to either $(U>0, V=0)$ or to $(U=$ $0, V>0)$. They correspond to the case where one and only one species survives. Once the semi-trivial solutions are constructed, global bifurcation techniques can be applied to obtain true coexistence solutions, i.e. solutions of the form $(U>0, V>0)$, from the semi-trivial ones,.

When the conservation law (1.3), is not aivalable, very few is known. Some perturbation results are available. In [13, the authors use a perturbation method to extend the above mentioned result when the equation $\sqrt{1.3}$ is nearly verified. Baxley and Robinson [16] study a very general system in the case of $N$ competing species, and they establish a result close to the bifurcation point.

In this paper, we propose a global method using the more general conservation equation

$$
\left(m_{0}(x)-a_{0} \Delta\right) R+\left(m_{1}(x)-a_{1} \Delta\right) U+\left(m_{2}(x)-a_{2} \Delta\right) V=I .
$$

Eliminating the unknow $R$ in 1.4 leads to nonlocal semi-trivial problems. We are able to study these semi-trivial problems by using a lower-upper solutions technique in the so-obtained scalar, nonlocal, elliptic equations. In an independent step, a specific use of global bifurcation techniques then allows to construct true coexistence solutions $(U>$ $0, V>0)$, starting from the semi-trivial solutions $(U>0, V=0)$ or $(U=0, V>0)$. This is a key step of our approach. We wish to stress that the lower-upper solutions part of our analysis requires (see Assumption 2 below) the crucial hypothesi: ${ }^{4}$

$$
\forall x \in \Omega, \quad \frac{m_{i}(x)}{a_{i}} \leq \frac{m_{0}(x)}{a_{0}} \quad(i=1,2) .
$$

It means that the ratio between death rate and diffusion rate should be larger for the resource than for the competing species, or, in other words, that the two species should diffuse relatively faster than the resource. Since spatial heterogeneity, and the associated diffusion processes, are the key to obtaining systems which allow coexistence, this assumption is quite natural: diffusion of the competing species helps obtaining coexistence situations. To be complete, let us mention that in the case when Robin boundary conditions are retained, another crucial assumption appears, namely

$$
\forall x \in \partial \Omega, \quad \frac{b_{i}(x)}{a_{i}} \leq \frac{b_{0}(x)}{a_{0}} \quad(i=1,2) .
$$

Assumption (1.6) is similar to 1.5 in spirit, in that a stronger ratio between the escape rate and the diffusion rate is required for the resource $R$ at the boundary, in comparison with the analogous ratio for populations $U$ and $V$.

The organization of the paper is as follows. In section 2 we present the notations and recall some technical results used in the paper. We also state our main results, namely Theorems 2.14 and 2.16. In section 3, we construct the above mentioned semi-trivial solutions. Under assumption 2, the lower-upper solutions method, in conjunction with bifurcation arguments, allows to prove existence, uniqueness, and non-degeneracy of the semi-trivial solutions. Section 4 is the main step of our study, in that we prove the

\footnotetext{
${ }^{4}$ In the case when the diffusion operators $a_{i} \Delta$ become $\operatorname{div} a_{i}(x) \nabla$ with $a_{i}(x)=\lambda_{i} a_{0}(x)$ $(i=1,2)$, the condition below becomes $m_{i}(x) / a_{i}(x) \leq m_{0}(x) / a_{0}(x)$ for $x \in \Omega(i=1,2)$.

5 This assumption obviously becomes $b_{i}(x) / a_{i}(x) \leq b_{0}(x) / a_{0}(x)$ for $x \in \partial \Omega(i=1,2)$, when the $a_{i}$ 's depend on $x$.
} 
existence of solutions $(R, U, V)$ to 1.2 that satisfy $R>0, U>0, V>0$. A global bifurcation theorem is used to construct these coexistence solutions, by joining the two families of semi-trivial solutions. Our construction leads to define a domain $\Theta \subset \mathbb{R}_{+}^{2}$ in the space of bifurcation parameters $\left(c_{1}, c_{2}\right)$, called the coexistence domain. This domain is such that whenever $\left(c_{1}, c_{2}\right) \in \Theta$, a coexistence solution is at hand. In section 5 , we state some consequences of our analysis, which provide an ecological point of view. Section 6 concludes this paper.

\section{Preliminaries and statement of our results}

\subsection{Generalities}

For $i=0,1,2$, the constants $a_{i}$ are supposed positive, and the fonctions $m_{i}(x)$ and $I(x)$ are assumed smooth, with $m_{i}(x)>0$ on $\bar{\Omega}$ and $I(x) \geq 0$ and $I(x) \not \equiv 0$ on $\bar{\Omega}$.

Taking a given $\alpha \in(0,1)$ whose value is irrelevant, we define the spaces ${ }^{6}$

$$
\begin{gathered}
X=\left\{u \in C^{2+\alpha}(\bar{\Omega}), \quad \partial_{n} u=0 \text { on } \partial \Omega\right\} \\
X_{+}=\{u \in X, \forall x \in \bar{\Omega}, u(x) \geq 0\}, \quad X_{+}^{*}=\left\{u \in X_{+}, \forall x \in \bar{\Omega}, u(x)>0\right\} .
\end{gathered}
$$

In the sequel, a solution to 1.2 is a triple $(R, U, V) \in X_{+}^{3}$ that satisfies 1.2. A coexistence solution is a solution that lies in $X_{+}^{*} \times X_{+}^{*} \times X_{+}^{*}$. For $i=0,1,2$, we note

$$
A_{i}:=m_{i}(x)-a_{i} \Delta .
$$

It is well known that, for all $\alpha \in(0,1)$, we have

$$
A_{i}:\left\{w \in C^{2+\alpha}(\Omega), \partial_{n} w=0 \text { on } \partial \Omega\right\} \longrightarrow C^{\alpha}(\Omega) \quad \text { is one-to-one. }
$$

In order to keep simple notations, the above operator will always be denoted by the same symbol $A_{i}$ for any choice of $\alpha$. In the similar spirit we note

$$
K_{i}:=A_{i}^{-1} \text {. }
$$

For each $i=0,1,2$, the operator $K_{i}$ is compact when seen as (more precisely : when extended to) an operator from $C^{1}(\Omega)$ to $C^{1}(\Omega)$ and from $L^{2}(\Omega)$ to $L^{2}(\Omega)$. Note that each operator $K_{i}$ maps $X$ to $X$ compactly as well. Recall that the strong maximum principle for elliptic operators with Neumann (or Robin) boundary conditions reads, whenever $u \in X$,

$$
\left\{\begin{array}{l}
A_{i} u \geq 0 \\
\partial_{n} u \geq 0 \\
u \neq \equiv 0
\end{array} \Longrightarrow \min _{x \in \bar{\Omega}} u(x)=m>0 .\right.
$$

The strong maximum principle also implies the following uniqueness

$$
\left\{\begin{array}{l}
A_{i} u=0 \\
\partial_{n} u=0
\end{array} \Longrightarrow u \equiv 0 .\right.
$$

We last recall the following standard Lemmas

\footnotetext{
6 with the obvious adaptation if Robin boundary conditions and/or variable coefficients $a_{i}$ 's are retained: to each operator $\operatorname{div} a_{i}(x) \nabla-m_{i}(x)$ with boundary condition $a_{i}(x) \partial_{n} \cdot+b_{i}(x) \cdot=0$ is associated the space $X_{i}=\left\{u \in C^{2+\alpha}(\bar{\Omega}), a_{i}(x) \partial_{n} u+b_{i}(x) u=0\right.$ on $\left.\partial \Omega\right\}$, and the triple $(R, U, V)$ then is to be exhibited in $X_{0,+} \times X_{1,+} \times X_{2,+}$.
} 
Lemma 2.1 Take $m(x) \in C^{\alpha}(\bar{\Omega})$ and $q(x) \in C^{\alpha}(\bar{\Omega})$. Assume $m(x)>0$ for all $x \in \bar{\Omega}$. Take $a \in \mathbb{R}_{+}^{*}$. Then the eigenvalue problem

$$
(m(x)-a \Delta) \phi+q(x) \phi=\lambda \phi \text { on } \Omega, \quad \partial_{n} \phi=0 \text { on } \partial \Omega
$$

has an infinite sequence of eigenvalues

$$
\lambda_{1}(q)<\lambda_{2}(q) \leq \ldots
$$

Moreover, $\lambda_{1}(q)=\min _{\phi \in H^{1}(\Omega), \phi \neq 0} \frac{\int a(\nabla \phi)^{2}+\int(m+q) \phi^{2}}{\int \phi^{2}}$ is a simple eigenvalue and the corresponding eigenfunction does not change sign on $\Omega$. The quantity $\lambda_{1}(q)$ is the only eigenvalue whose associated eigenfunction does not change sign on $\Omega$. Finally $\lambda_{1}(q)$ depends continuously on $q$ and, if $q_{1} \leq q_{2}$ with $q_{1} \neq q_{2}$, then $\lambda_{1}\left(q_{1}\right)<\lambda_{1}\left(q_{2}\right)$.

Lemma 2.2 Take $q(x) \in C^{\alpha}(\bar{\Omega}), a \in \mathbb{R}_{+}^{*}$ such that $q(x)>0$ for any $x \in \bar{\Omega}$. Then the eigenvalue problem

$$
(m(x)-a \Delta) \phi=\mu q(x) \phi, \quad \partial_{n} \phi=0,
$$

has an infinite sequence of eigenvalues

$$
0<\mu_{1}(q)<\mu_{2}(q) \leq \ldots
$$

Moreover, $\mu_{1}(q)=\min _{\phi \in H^{1}(\Omega), \phi \neq 0} \frac{\int a(\nabla \phi)^{2}+\int m \phi^{2}}{\int q \phi^{2}}$ is a simple eigenvalue and the corresponding eigenfunction does not change sign on $\Omega$. The quantity $\mu_{1}(q)$ is the only eigenvalue whose associated eigenfunction does not change sign on $\Omega$. Moreover, $\mu_{1}(q)$ depends continuously on $q$ and, if $q_{1} \leq q_{2}$ with $q_{1} \neq q_{2}$, then $\mu_{1}\left(q_{1}\right)<\mu_{1}\left(q_{2}\right)$.

\subsection{Lower- and upper-solutions}

In order to make use of a lower-upper solution technique later in this text, we readily introduce the following assumption

Assumption 1 For $i=1,2$, we assume $f_{i}(x, R) \in C^{1}(\bar{\Omega} \times \mathbb{R})$, with $f_{1}(x, R) \leq 0$ whenever $R \leq \mathrm{d}$. Besides, we assume that for any $x \in \bar{\Omega}$, we have

$$
\forall R>0, \quad f_{i}(x, R)>0, \quad \text { and } \quad \frac{\partial f_{i}}{\partial R}(x, R)>0 .
$$

In other words, the consumption rate is supposed to be non-negative and increasing function of the resource. We also introduce the following crucial one-sided condition 8

Assumption 2 For $i=1,2$ and $x \in \Omega$, we have

$$
m_{i}(x) / a_{i} \leq m_{0}(x) / a_{0} .
$$

7 Recall that we are only interested in situations with $R \geq 0$, hence the way we extend $f_{i}$ for negative values of $R$ is irrelevant.

8 See footnote 4 in the case of variable coefficients diffusion operators. 
As we show now, this condition provides a monotonicity property that plays a key rôle in our analysis. Whenever $w \in X_{+}$, define $R_{i}(w) \in X$ as the unique solution in $X$ to

$$
A_{0} R_{i}(w)+A_{i} w=I
$$

The operator $w \mapsto R_{i}(w)$ is introduced for the following reason. The one-species problem (corresponding to semi-trivial solutions $(U>0, V=0)$ say), reads

$$
A_{0} R+c_{1} f_{1}(x, R) U=I, \quad A_{1} U-c_{1} f_{1}(x, R) U=0 .
$$

This in turn is equivalent to

$$
R=R_{1}(U), \quad A_{1} U-c_{1} f_{1}\left(x, R_{1}(U)\right) U=0,
$$

and $R_{1}(U)$ may be seen as the resource at hand in the presence of the population $U$. In any circumstance, the one-species problem leads to considering the above nonlinear and nonlocal elliptic problem, with nonlinearity $w \mapsto f_{1}\left(R_{1}(w)\right) w$.

Now, an easy computation provides the alternative formula 9

$$
R_{i}(w)=K_{0}(I)-\frac{a_{i}}{a_{0}} K_{0} A_{0} w+\frac{1}{a_{0}} K_{0}\left(\left(a_{i} m_{0}(x)-a_{0} m_{i}(x)\right) w\right) .
$$

A key point is the fact that the nonlocal term $K_{0}\left(a_{i} m_{0}-a_{0} m_{i}\right) w$ above satisfies

$$
K_{0}\left(a_{i} m_{0}-a_{0} m_{i}\right) w \geq 0 \text { whenever } w \geq 0,
$$

as an obvious consequence of Assumption 2 together with the maximum principle. Another remark is in order. In the case of Neumann boundary conditions, we have the obvious relation $K_{0} A_{0} w=w$. The reader's attention is drawn to the fact that in the case of Robin boundary condition, we have $K_{0} A_{0} w \neq w$ in general. Note however that the following holds. Provided we assume $b_{i} / a_{i} \leq b_{0} / a_{0}(i=1,2)-$ see equation (1.6) and footnote 5- we have

$$
K_{0} A_{0} w \leq w \text { whenever } w \geq 0
$$

This comes from the maximum principle together with the fact that, when $w \geq 0$, the function $v=K_{0} A_{0} w$ satisfies $A_{0}(v-w)=0$ with the boundary condition $\left(a_{0} \partial_{n}+\right.$ $\left.b_{0}\right)(v-w)=+\left(a_{0} b_{1}-b_{0} a_{1}\right) w / a_{1} \leq 0$.

We readily show that Assumption 2 implies the following one-sided Lipschitz condition for the nonlinearity $w \mapsto f_{1}\left(R_{1}(w)\right) w$ in 2.8 .

Lemma 2.3 Suppose Assumption 2 is true. Let $M$ be a positive constant and take $i=1,2$. Then, there exists $\gamma=\gamma_{i}(M)>0$ such that

$$
w_{1}(x) f_{i}\left(x, R_{i}\left(w_{1}\right)\right)(x)-w_{2}(x) f_{i}\left(x, R_{i}\left(w_{2}\right)\right)(x) \geq-\gamma\left(w_{1}(x)-w_{2}(x)\right)
$$

whenever $w_{1}, w_{2} \in X$ satisfy $0 \leq w_{2} \leq w_{1} \leq M$.

Remark 2.4 The point is, the above estimate is pointwise in $x$, though it involves the nonlocal operator $R_{i}$.

${ }^{9}$ When the diffusion operators become $\operatorname{div} a_{i}(x) \nabla$ with $a_{i}(x)=\lambda_{i} a_{0}(x)$, see footnotes 2 and 4 . the formula below becomes $R_{i}(w)=K_{0}(I)-\lambda_{i} K_{0} A_{0} w+K_{0}\left(\left(\lambda_{i} m_{0}(x)-m_{1}(x)\right) w\right)$, with $\lambda_{i} m_{0}(x)-m_{1}(x) \geq 0$ for all $x$, and our analysis is unchanged. 
Remark 2.5 If all diffusion operators are the same, as in the previously quoted papers, namely if $A_{i} \equiv A_{0}(i=1,2)$, then the nonlocal terms of the form $K_{0}\left(a_{i} m_{0}-a_{0} m_{i}\right) w$ vanish in the course of the analysis. In that particular case, the method we develop coincides with that of 18 . The nonlocal terms constitute the main difficulty we treat.

Admitting Lemma 2.3 is proved for the moment, we readily state that this result allows us to apply a lower-upper solution method in the nonlocal elliptic system

$$
A_{i} w-c_{i} f_{i}\left(x, R_{i}(w)(x)\right) w=0,
$$

where $w \in X$ is the unknown. Indeed, using Lemma 2.3 the following definition and Theorem are standard (see [5]).

Definition 2.6 (lower- and upper-solutions) An upper-solution to equation 2.12 is a function $w \in C^{2+\alpha}(\bar{\Omega})$ verifying ${ }^{10}$

$$
A_{i} w(x)-c_{i} f_{i}\left(x, R_{i}(w)(x)\right) w(x) \geq 0 \text { for all } x \in \Omega, \quad \text { and } \partial_{n} W \geq 0 \text { on } \partial \Omega .
$$

A lower-solution is defined in the similar way with reversed inequalities.

\section{Theorem 2.7 (lower-upper solutions method - See [5])}

Assume there exists a lower resp. upper solution $W^{1}$ resp. $W^{2}$ to equation (2.12), which satisfies $0 \leq W^{1} \leq W^{2}$.

Then, equation 2.12 admits a pair $\left(W^{-}, W^{+}\right)$of solutions, with $W^{1} \leq W^{-} \leq$ $W^{+} \leq W^{2}$

$\bar{\Omega}$

If $W^{1}$ and $W^{2}$ are not solutions to 2.12, we have $W^{1}<W^{-} \leq W^{+}<W^{2}$ on

The pair $\left(W^{-}, W^{+}\right)$is maximal in the sense that each solution $W$ to 2.12 which satisfies $W \in\left[W^{1}, W^{2}\right]$ necessarily verifies $W \in\left[W^{-}, W^{+}\right]$as well.

Remark 2.8 Stricto sensu the above Theorem is not to be found in [5]. Smoller requires the nonlinear term be Lipschitz in $w$, a property that we do not have at hand in the present case. It is standard to observe that the key of the proof, which relies on an iteration of the maximum principle, is the following. When writing the equation $A_{i} w=c_{i} f_{i}\left(x, R_{i}(w)\right) w=: G_{i}(x, w)$, the point is to find a (large) $K>0$ and a (large) $M>0$ such that whenever $0 \leq W_{1}(x) \leq W_{2}(x) \leq M$ for all $x$, we have $G\left(x, W_{1}\right)(x)+K W_{1}(x) \leq G\left(x, W_{2}\right)(x)+K W_{2}(x)$ for all $x$ as well. The one-sided Lipschitz estimate of Lemma 2.3 is enough in that respect.

Note that Pao [7,6] establishes variants of the above techniques for systems, in the case where the nonlinear terms, which are vector-valued, satisfy so-called quasimonotonicity properties.

There remains to prove Lemma 2.3

Proof of Lemma 2.3

Firstly, when $w \in X$ satisfies $0 \leq w \leq M$, the maximum principle provides in 2.9 )

$$
\left\|R_{i}(w)\right\|_{L^{\infty}} \leq\left\|K_{0}(I)\right\|_{L^{\infty}}+M\left\|K_{0}\left(m_{0}\right)\right\|_{L^{\infty}}+\frac{M}{a_{0}}\left\|K_{0}\left(a_{i} m_{0}-a_{0} m_{i}\right)\right\|_{L^{\infty}}=: M_{\infty} .
$$

$\overline{10}$ With the obvious extension in the case of Robin boundary conditions. 
The assumed smoothness of $f_{i}$ ensures that $f_{i}$ is globally Lipschitz on $\bar{\Omega} \times\left[-M_{\infty}, M_{\infty}\right]$. We call $C_{i}$ the Lipstchitz constant associated with $f_{i}$.

Next, whenever $0 \leq w_{2} \leq w_{1} \leq M$, with $w_{i} \in X(i=1,2)$, we have

$$
\begin{aligned}
R_{i}\left(w_{1}\right)-R_{i}\left(w_{2}\right) & =-\frac{a_{i}}{a_{0}} K_{0} A_{0}\left(w_{1}-w_{2}\right)+\frac{1}{a_{0}} K_{0}\left(\left(a_{i} m_{0}(x)-a_{0} m_{i}(x)\right)\left(w_{1}-w_{2}\right)\right) \\
& \geq-\frac{a_{i}}{a_{0}} K_{0} A_{0}\left(w_{1}-w_{2}\right) \\
& \geq-\frac{a_{i}}{a_{0}}\left(w_{1}-w_{2}\right) .
\end{aligned}
$$

where the first lower bound uses Assumption 2 while the second uses the observation 2.10. Hence, writing

$$
\begin{aligned}
& w_{1}(x) f_{i}\left(x, R_{i}\left(w_{1}\right)(x)\right)-w_{2}(x) f_{i}\left(x, R_{i}\left(w_{2}\right)(x)\right) \\
& \quad=f_{i}\left(x, R_{i}\left(w_{1}\right)(x)\right)\left(w_{1}-w_{2}\right)(x)+w_{2}(x)\left(f_{i}\left(x, R_{i}\left(w_{1}\right)(x)\right)-f_{i}\left(x, R_{i}\left(w_{2}\right)(x)\right)\right) \\
& \quad \geq w_{2}(x)\left(f_{i}\left(x, R_{i}\left(w_{1}\right)(x)\right)-f_{i}\left(R_{i}\left(x, w_{2}\right)(x)\right)\right)
\end{aligned}
$$

we distinguish two cases. If $x$ is such that $R_{i}\left(w_{1}\right)(x) \geq R_{i}\left(w_{2}\right)(x)$, then $f_{i}$ being an increasing function of $R$, we recover

$$
w_{1}(x) f_{i}\left(x, R_{i}\left(w_{1}\right)(x)\right)-w_{2}(x) f_{i}\left(x, R_{i}\left(w_{2}\right)(x)\right) \geq 0 .
$$

In the opposite case we have

$$
\begin{aligned}
w_{1}(x) & f_{i}\left(x, R_{i}\left(w_{1}\right)(x)\right)-w_{2}(x) f_{i}\left(x, R_{i}\left(w_{2}\right)(x)\right) \\
& \geq w_{2}(x)\left(f_{i}\left(x, R_{i}\left(w_{1}\right)(x)\right)-f_{i}\left(R_{i}\left(x, w_{2}\right)(x)\right)\right) \\
& \geq+C_{i} w_{2}(x)\left(R_{i}\left(w_{1}\right)(x)-R_{i}\left(w_{2}\right)(x)\right) \\
& \geq-C_{i} \frac{a_{i}}{a_{0}} w_{2}(x)\left(w_{1}(x)-w_{2}(x)\right) \geq-C_{i} \frac{a_{i}}{a_{0}} M\left(w_{1}(x)-w_{2}(x)\right) .
\end{aligned}
$$

The proposition is proved.

2.3 Bifurcation methods

We state the two bifurcation theorems we use in the sequel; for equations of the form,

$$
T(c, W)=W
$$

where $c \in \mathbb{R}$ is the bifurcation parameter, $W \in Y$ is the seeked solution, and $Y$ is a Banach space, while $T(c, W) \in C^{0}(\mathbb{R} \times Y ; Y)$ is a given, continuous map. In the following we assume that $T$ is twice Fréchet-differentiable in $(c, W)$, and we denote by $D_{c}$ resp. $D_{W}$ the Fréchet derivatives of $T$ with respect to $c$ resp. $W$.

We start with the local bifurcation theorem of Crandall-Rabinowitz [1].

Theorem 2.9 (Local bifurcation from a simple eigenvalue - see [1])

With the above notation, we assume that

$$
\forall c \in \mathbb{R}, \quad T(c, 0)=0 .
$$


We also assume that for some value $c^{0} \in \mathbb{R}$, the following holds:

$$
\left\{\begin{array}{l}
\operatorname{dim}\left(\operatorname{Ker}\left(L\left(c^{0}\right)\right)=1, \quad \text { where we note } L(c)=\operatorname{Id}-D_{W} T(c, 0),\right. \\
\operatorname{codim}\left(\operatorname{Im}\left(L\left(c^{0}\right)\right)\right)=1, \\
\text { and, whenever } W_{0} \text { satisfies } \operatorname{Ker}\left(L\left(c^{0}\right)\right)=\operatorname{span}\left(W_{0}\right) \text {, we have } \\
\quad D_{c} L\left(c^{0}\right) \cdot W_{0} \notin \operatorname{Im}\left(L_{0}\right) .
\end{array}\right.
$$

Then, there exists $\varepsilon>0$ and a map $(c(s), X(s)) \in C^{0}((-\varepsilon, \varepsilon) ; \mathbb{R} \times Y)$, with $c(0)=c^{0}$, $X(0)=0$, such that close to $\left(c^{0}, 0\right)$ in $\mathbb{R} \times Y$, the only nontrivial solution to $T(c, W)=$ $W$ is given by

$$
\left\{\begin{array}{l}
T(c, W)=W \\
(c, W) \neq(c, 0)
\end{array} \Longleftrightarrow \exists s \in(-\varepsilon, \varepsilon) \text { such that }(c, W)=\left(c(s), s W_{0}+s X(s)\right) .\right.
$$

We complete the picture by stating a global version of the theorem. Some additional assumptions are required. We need the following compactness assumption

$$
\begin{aligned}
& T: \mathbb{R} \times Y \rightarrow Y \text { is a compact operator, and, } \\
& \forall(c, W), \quad T(c, W)=D_{W} T(c, 0) \cdot W+\mathcal{R}(c, W),
\end{aligned}
$$

where $D_{W} T(c, 0)$ is a linear compact operator.

In other words we assume that the linearized part of equation $T(c, W)=W$, close to the trivial solution $W=0$, is always a compact perturbation of the identity.

Now, for those values of $c$ such that the trivial solution $W=0$ is an isolated solution to $T(c, W)=W$, i.e. typically whenever $D_{W} T(c, 0)$ does not admit 1 as an eigenvalue, one may define the index of the solution $W=0$, as the Leray-Schauder degree $\operatorname{deg}(\operatorname{Id}-T(c, \cdot), B, 0)$ (here $B \subset Y$ is a ball centred at 0 such that $W=0$ is the only solution to $T(c, W)=W$ in $B)$. In other words, the index of the considered solution $W=0$ is

$$
i(T(c, \cdot), 0):=\operatorname{deg}(\operatorname{Id}-T(c, \cdot), B, 0) .
$$

It has the value

$$
i(T(c, \cdot), 0)=\operatorname{deg}\left(\operatorname{Id}-D_{W} T(c, 0), B, 0\right)=(-1)^{p},
$$

where $p$ is the sum of the algebraic multiplicities of all (real) eigenvalues of $D_{W} T(c, 0)$ that are greater than 1 .

The following theorem holds true

Theorem 2.10 (Global bifurcation from a simple eigenvalue - see 20,18 ) Under the assumptions and notation of Theorem 2.9, we suppose that $T$ is a compact operator such that $D_{W} T(c, 0)$ is linear compact for any $c$, as in 2.13 .

We also assum $\varepsilon^{11}$ that for some $\varepsilon>0$, the index $i(T(c, \cdot), 0)$ is constant on $\left(c_{0}-\right.$ $\left.\varepsilon, c_{0}\right)$ and on $\left(c_{0}, c_{0}+\varepsilon\right)$, and that whenever $c_{0}-\varepsilon<\alpha<c_{0}<\beta<c_{0}+\varepsilon$ we have $i(T(\alpha, \cdot), 0) \neq i(T(\beta, \cdot), 0)$.

\footnotetext{
11 This second assumption is not needed when $D_{c} T(c, 0)$ does not depend on $c$. In our case see below - we shall apply this Theorem for T's of the form $T(c, W)=A+c B(W)$ where $A$ is a constant and $B$ a compact operator independent of $c$. This is due to our choice of bifurcation parameters: they are only involved in the two terms $c_{1} f_{1}(R) U$ and $c_{2} f_{2}(R) V$ in 1.2 , terms which are proportional with $c_{1}$ resp. $c_{2}$. We nevertheless describe our bifurcation method in the present more general form, in order to keep a procedure that applies as well in the case of a nonlinear dependence on the bifurcation parameters, as would be the case when choosing $\left(c_{1}, c_{2}\right)$-dependent consumption rates for instance.
} 
Then, there exists a continuum $\mathrm{L}^{12} \mathscr{C}$ of nontrivial solutions to $T(c, W)=W$ in $\mathbb{R} \times Y$ such that one of the following alternatives holds:

(i) The closure $\overline{\mathscr{C}}$ joins the trivial solution $\left(c^{0}, 0\right)$ to another trivial solution $(\widehat{c}, 0)$, for some $\widehat{c} \in \mathbb{R}, \widehat{c} \neq c_{0}$, where $\operatorname{Id}-D_{W} T(\widehat{c}, 0)$ is not invertible.

(ii) The closure $\overline{\mathscr{C}}$ joins $\left(c_{0}, 0\right)$ to $\infty$ in $\mathbb{R} \times Y$.

2.4 Statement of our results

Our whole construction relies on a recursive procedure. We construct coexistence solutions to 1.2 (we do not rewrite the boundary conditions),

$$
\left\{\begin{array}{l}
A_{0} R+c_{1} f_{1}(x, R) U+c_{2} f_{2}(x, R) V=I \\
A_{1} U-c_{1} f_{1}(x, R) U=0 \\
A_{2} V-c_{2} f_{2}(x, R) V=0
\end{array}\right.
$$

by starting from the 0 -species problem (namely trivial solutions corresponding to $R>$ $0, U=0, V=0)$,. Then we construct 1-species, or semi-trivial, solutions (corresponding to $R>0$, and either $(U>0, V=0)$ or $(U=0, V>0)$ ), by using lower-upper solutions techniques. This step is complemented with the use of bifurcations from the 0 -species problem, to prove the non-degeneracy of the so-obtained semi-trivial solutions, and to compute the index of these solutions. This step is crucial, and makes a strong use of our Assumption 2, It is the most difficult and technical part of our analysis. Armed with these results, we then use bifurcations again to construct true coexistence solutions $R>0, U>0, V>0$. This last step uses all informations gathered on the semi-trivial solutions.

We start with the 0 -species problem.

Theorem 2.11 (Trivial solution)

(i) The following equation has a unique solution $S \in X_{+}^{*}$,

$$
A_{0} S=I
$$

(ii) If $(R, U, V) \in X_{+}^{3}$ is a solution to 1.2 with $U \not \equiv 0$ or $V \not \equiv 0$, ther ${ }^{13} 0<R<S$.

(iii) Let $w \in X_{+}$. The equation

$$
A_{0} R+c_{i} f_{i}(x, R) w=I
$$

has a unique solution ${ }^{14} R_{w}^{(i)} \in X_{+}^{*}$. It satisfies $0<R_{w}^{(i)} \leq S$. The map $w \mapsto R_{w}^{(i)}$ is decreasing from $X_{+}$to $X_{+}$.

12 We call a continuum of solutions a connected familly of solutions $(c, W) \in R \times Y$.

13 Recall that throughout this text the notation $R<S$ means $S-R \in X_{+}^{*}$, or, in other words, that for any $x \in \bar{\Omega}$ we have $R(x)<S(x)$

${ }^{14}$ Note that $R_{w}^{(i)} \neq R_{i}(w)$, see 2.9 , unless we have $A_{i} w=c_{i} f_{i}\left(x, R_{w}^{(i)}\right) w$. 
We postpone the (easy) proof of this statement.

We next focus our attention on semi-trivial solutions to $(1.2)$.

If $V \equiv 0$ (the case $U \equiv 0$ is similar), system 1.2 reduces to (we do not rewrite the boundary conditions)

$$
\left\{\begin{array}{l}
A_{0} R+c_{1} f_{1}(x, R) U=I, \\
A_{1} U-c_{1} f_{1}(x, R) U=0 .
\end{array}\right.
$$

We define the operator

$$
T_{1}\left(c_{1}, R, U\right)=\left(\begin{array}{c}
K_{0}\left(I-c_{1} f_{1}(x, R) U\right) \\
K_{1}\left(c_{1} f_{1}(x, R) U\right)
\end{array}\right)=\left(\begin{array}{c}
S \\
0
\end{array}\right)+c_{1}\left(\begin{array}{c}
-K_{0}\left(f_{1}(x, R) U\right) \\
K_{1}\left(f_{1}(x, R) U\right)
\end{array}\right) .
$$

Clearly, $T_{1}: \mathbb{R} \times X^{2} \rightarrow X^{2}$ is continuous and compact, any fixed point $(R, U) \in X^{2}$ of $T_{1}\left(c_{1}, \cdot, \cdot\right)$, i.e. such that $T_{1}\left(c_{1}, R, U\right)={ }^{t}(R, U)$, is clearly a solution to (2.18), and the trivial solution is $T_{1}\left(c_{1}, S, 0\right)={ }^{t}\left(c_{1}, S, 0\right)$.

The following theorem describes two solution branches to 2.18 . It is proved in section 4, using a global bifurcation technique with $c_{1}$ used as the bifurcation parameter.

Theorem 2.12 (Semi-trivial solutions)

Under Assumptions 1 and 2 , the following holds.

(i) There exists $c_{1}^{0}>0$ such that:

- if $c_{1} \leq c_{1}^{0}$, then $(S, 0)$ is the only solution to 2.18 in $X_{+}^{2}$,

- if $c_{1}>c_{1}^{0}$, the system 2.18 has a unique solution in $\left(X_{+}^{*}\right)^{2}$, noted

$$
\left(R_{u}^{*}\left(c_{1}\right), U^{*}\left(c_{1}\right)\right)
$$

(ii) Whenever $c_{1}>c_{1}^{0}$, the solution $\left(R_{u}^{*}\left(c_{1}\right), U^{*}\left(c_{1}\right)\right) \in\left(X_{+}^{*}\right)^{2}$ is non-degenerat $\overline{15}$ and ${ }^{16} i\left(T_{1}\left(c_{1}, \cdot\right),\left(R_{u}^{*}\left(c_{1}\right), U^{*}\left(c_{1}\right)\right)\right)=1^{17}$

(iii) The map $R_{u}^{*}: c_{1} \mapsto R_{u}^{*}\left(c_{1}\right)$ is decreasing, and belongs to $C^{1}\left(\left(c_{1}^{0},+\infty\right), X_{+}^{*}\right)$. Moreover, the following two limits hold uniformly on $\bar{\Omega}$, namely,

$$
R_{u}^{*}\left(c_{1}\right) \underset{c_{1} \rightarrow c_{1}^{0}}{\longrightarrow} S, \quad \text { and } R_{u}^{*}\left(c_{1}\right) \underset{c_{1} \rightarrow+\infty}{\longrightarrow} 0
$$

(iv) The map $U^{*}: c_{1} \mapsto U^{*}\left(c_{1}\right)$ is increasing, and belongs to $C^{1}\left(\left(c_{1}^{0},+\infty\right), X_{+}^{*}\right)$. Moreover, the following two limits hold uniformly on $\bar{\Omega}$, namely,

$$
U^{*}\left(c_{1}\right) \underset{c_{1} \rightarrow c_{1}^{0}}{\longrightarrow} 0, \quad \text { and } U^{*}\left(c_{1}\right) \underset{c_{1} \rightarrow+\infty}{\longrightarrow} U_{\infty}
$$

where $U_{\infty} \in X_{+}^{*}$ is the unique solution to $A_{1} U_{\infty}=I$.

15 In other words, $\operatorname{Ker}\left(\operatorname{Id}-D_{(R, U)} T_{1}\left(c_{1}, R_{u}^{*}\left(c_{1}\right), U^{*}\left(c_{1}\right)\right)\right)=\{0\}$.

16 Our proof not only provides that the index of this solution $\left(R_{u}^{*}\left(c_{1}\right), U^{*}\left(c_{1}\right)\right)$ is one, but also that all eigenvalues of $\mathrm{Id}-D_{(R, U)} T_{1}\left(c_{1}, R_{u}^{*}\left(c_{1}\right), U^{*}\left(c_{1}\right)\right)$ are less than one whenever $c_{1}>c_{1}^{0}$ is close to $c_{1}^{0}$. This implies that the so-obtained solution is stable, i.e. the associated time-dependent parabolic problem admits $\left(R_{u}^{*}\left(c_{1}\right), U^{*}\left(c_{1}\right)\right)$ as a locally stable steady state.

17 This apparently technical statement is the key to constructing true coexistence solutions and obtaining Theorem 2.14 below. 
Remark 2.13 In fact, the mere existence of semi-trivial solutions may be obtained using a simple global bifurcation argument, without making use of our Assumption 2 Assumption 2 is required at variance to obtain uniqueness of these solutions. This assumption also plays a key rôle to establish non-degeneracy, and to compute the value of the index.

Naturally, the similar results hold in the case $U \equiv 0$ and $V>0$. This provides a critical value $c_{2}^{0}$, and a solution branch $\left(R_{v}^{*}\left(c_{2}\right), V^{*}\left(c_{2}\right)\right) \in\left(X_{+}^{*}\right)^{2}$ whenever $c_{2}>c_{2}^{0}$, which satisfies the properties similar to the ones listed before. The natural semi-trivial solutions to 1.2 are $(R, U, V)=\left(R_{u}^{*}\left(c_{1}\right), U^{*}\left(c_{1}\right), 0\right)$ (with $\left.c_{1}>c_{1}^{0}\right)$, and $(R, U, V)=$ $\left(R_{v}^{*}\left(c_{2}\right), 0, V^{*}\left(c_{2}\right)\right)$ (with $\left.c_{2}>c_{2}^{0}\right)$. We define the following two subsets of $\mathbb{R}_{+}^{2} \times X_{+}^{3}$, namely

$$
\begin{aligned}
& \mathscr{C}_{u}=\left\{\left(c_{1}, c_{2}, R_{u}^{*}\left(c_{1}\right), U^{*}\left(c_{1}\right), 0\right) ; c_{1}>c_{1}^{0}\right\}, \\
& \mathscr{C}_{v}=\left\{\left(c_{1}, c_{2}, R_{v}^{*}\left(c_{2}\right), 0, V^{*}\left(c_{2}\right)\right) ; c_{2}>c_{2}^{0}\right\} .
\end{aligned}
$$

With this notation at hand, the following Theorem is the main result of the present paper. It establishes that coexistence solutions to 1.2 may be defined using bifurcations from the two sets $\mathscr{C}_{u}$ and $\mathscr{C}_{v}$. The proof is provided in section 5.2 Figure 2.1 illustrates the situation.

Theorem 2.14 (Coexistence solutions)

Under Assumptions 1 and 2 , the following holds.

(i) (Bifurcations from $\mathscr{C}_{u}$ to $\mathscr{C}_{v}$ ). Let $c_{1}>c_{1}^{0}$ be fixed.

There exist $c_{2}^{*}=c_{2}^{*}\left(c_{1}\right)>c_{2}^{0}$ and $c_{2}^{* *}=c_{2}^{* *}\left(c_{1}\right)>c_{2}^{0}$, and there is a continuum of positive solutions to 1.2 , noted $\left(c_{1}, c_{2}, R, U, V\right) \in\left(c_{1}^{0},+\infty\right) \times\left(c_{2}^{0},+\infty\right) \times\left(X_{+}^{*}\right)^{3}$, whose closure joins the semi-trivial $\left(c_{1}, c_{2}^{*}, R_{u}^{*}\left(c_{1}\right), U^{*}\left(c_{1}\right), 0\right) \in \mathscr{C} u$ to the semi-trivial $\left(c_{1}, c_{2}^{* *}, R_{v}^{*}\left(c_{2}^{* *}\right), 0, V^{*}\left(c_{2}^{* *}\right)\right) \in \mathscr{C}_{v}$.

In particular, noting $\underline{c_{2}}\left(c_{1}\right)=\min \left(\left\{c_{2}^{*}, c_{2}^{* *}\right\}\right) \leq \max \left(\left\{c_{2}^{*}, c_{2}^{* *}\right\}\right)=\overline{c_{2}}\left(c_{1}\right)$, we have

$\forall c_{2} \in\left(\underline{c_{2}}\left(c_{1}\right), \overline{c_{2}}\left(c_{1}\right)\right), \quad \exists(R, U, V) \in\left(X_{+}^{*}\right)^{3}$ coexistence solution to 1.2 .

(ii) (Bifurcations from $\mathscr{C}_{v}$ to $\mathscr{C}_{u}$ ). Let $c_{2}>c_{2}^{0}$ be fixed.

There exist $c_{1}^{*}=c_{1}^{*}\left(c_{2}\right)>c_{1}^{0}$ and $c_{1}^{* *}=c_{1}^{* *}\left(c_{2}\right)>c_{1}^{0}$, and there is a continuum of positive solutions to 1.2 , noted $\left(c_{1}, c_{2}, R, U, V\right) \in\left(c_{1}^{0},+\infty\right) \times\left(c_{2}^{0},+\infty\right) \times\left(X_{+}^{*}\right)^{3}$, whose closure joins the semi-trivial $\left(c_{1}^{*}, c_{2}, R_{v}^{*}\left(c_{2}\right), 0, V^{*}\left(c_{2}\right)\right) \in \mathscr{C}_{v}$ to the semi-trivial $\left(c_{1}^{* *}, c_{2}, R_{u}^{*}\left(c_{1}^{* *}\right), U^{*}\left(c_{1}^{* *}\right), 0\right) \in \mathscr{C}_{u}$.

In particular, noting $\underline{c_{1}}\left(c_{2}\right)=\min \left(\left\{c_{1}^{*}, c_{1}^{* *}\right\}\right) \leq \max \left(\left\{c_{1}^{*}, c_{1}^{* *}\right\}\right)=\overline{c_{1}}\left(c_{2}\right)$, we have

$$
\forall c_{1} \in\left(\underline{c_{1}}\left(c_{2}\right), \overline{c_{1}}\left(c_{2}\right)\right), \quad \exists(R, U, V) \in\left(X_{+}^{*}\right)^{3} \text { coexistence solution to } 1.2 \text {. }
$$

Remark 2.15 Note that the situation where $c_{2}^{*}\left(c_{1}\right)=c_{2}^{* *}\left(c_{1}\right)$, say, may very well happen. In that case the interval $\left(\underline{c_{1}}\left(c_{2}\right), \overline{c_{1}}\left(c_{2}\right)\right)$ is void. Hence, as we can see, the second statement in part (i) of the Theorem is a weak byproduct of the first one, which exhibits at variance an actual branch of coexistence solutions. We refer to the conjecture stated in paragraph 6 below for a discussion of this point. 


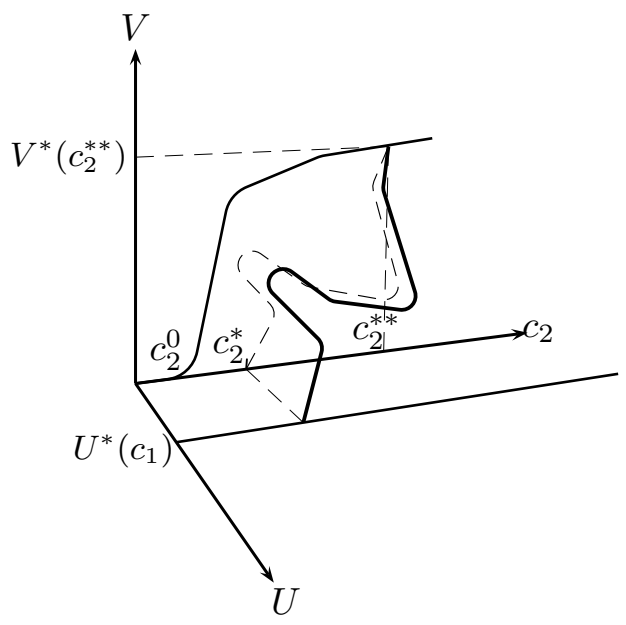

Fig. 2.1 Coexistence solution in the space $\mathbb{R} \times X_{+} \times X_{+}$.

The parameter $c_{1}$ is fixed here, with $c_{1}>c_{1}^{0}$.

Dashed lines in the $\left(U, c_{2}\right)$-plane represent (the projection of) few semi-trivial solutions $(R, U, 0)$ in this plane: due to their very definition, these solutions do not depend on $c_{2}$. The particular semi-trivial solution associated with $U^{*}\left(c_{1}\right)$ - see Theorem 2.12 - is represented by a full line. The full curve in the $\left(c_{2}, V\right)$-plane represents the (projection of the) family of semi-trivial solutions $\left(R, 0, V^{*}\left(c_{2}\right)\right)$. Finally, the bold curve joining the two planes $\left(c_{2}, U\right)$ and $\left(c_{2}, V\right)$ represents the (projection of the) coexistence solutions $\left(c_{1}, c_{2}, R, U, V\right) \in$ $\left(c_{1}^{0}, \infty\right) \times\left(\underline{c_{2}}\left(c_{1}\right), \overline{c_{2}}\left(c_{1}\right)\right) \times\left(X_{+}^{*}\right)^{3}$ obtained in part (i) of the Theorem. In the present figure we have assumed $c_{2}^{*}\left(c_{1}\right)<c_{2}^{* *}\left(c_{1}\right)$.

With the use of the above Theorem, one may define a coexistence domain $\Theta$, as

$$
\begin{aligned}
& \Theta=\left\{\left(c_{1}, c_{2}\right) \in\left(c_{1}^{0},+\infty\right) \times\left(c_{2}^{0},+\infty\right),\right. \text { s.t. } \\
& \left.\qquad c_{1} \in\left(\underline{c_{1}}\left(c_{2}\right), \overline{c_{1}}\left(c_{2}\right)\right) \text { and } c_{2} \in\left(\underline{c_{2}}\left(c_{1}\right), \overline{c_{2}}\left(c_{1}\right)\right)\right\} .
\end{aligned}
$$

It corresponds to values of the parameters $\left(c_{1}, c_{2}\right)$ for which a coexistence solution may be exhibited (a subset of the set of all values $\left(c_{1}, c_{2}\right)$ such that a coexistence solution may be exhibited - see paragraph 6 on that point).

The following Theorem is proved in section 5.3. It explores the structure of $\Theta$.

Theorem 2.16 (Coexistence domain)

Under Assumption 1 and 2, and with the notation of Theorem 2.14, the following holds.

(i) Whenever $c_{1}>c_{1}^{0}$, the quantity $c_{2}^{* *}\left(c_{1}\right)$ is characterised by the relation

$$
c_{1}^{*}\left(c_{2}^{* *}\left(c_{1}\right)\right)=c_{1},
$$

and similarly when indices 1 and 2 are reversed.

(ii) The two maps

$$
c_{1}^{*}\left(c_{2}\right):\left(c_{2}^{0},+\infty\right) \longrightarrow\left(c_{1}^{0},+\infty\right), \quad \text { and } \quad c_{2}^{*}\left(c_{1}\right):\left(c_{1}^{0},+\infty\right) \longrightarrow\left(c_{2}^{0},+\infty\right)
$$




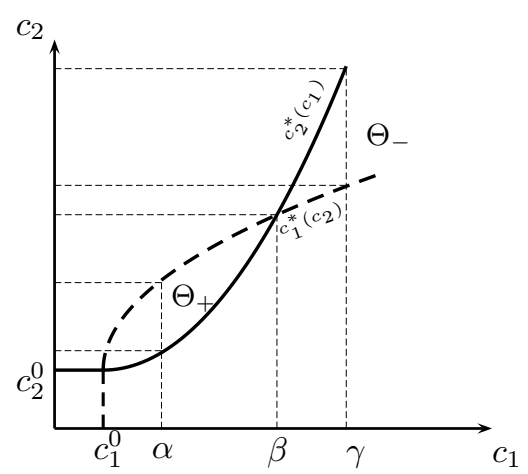

(a) Coexistence domain



(b) Bifurcation curves

Fig. 2.2 Coexistence domain and bifurcation solutions.

Figure (a) shows a possible coexistence domain $\Theta$. The full curve represents $\left(c_{1}, c_{2}^{*}\left(c_{1}\right)\right)$ and the dashed one represents $\left(c_{1}^{*}\left(c_{2}\right), c_{2}\right)$. For any $t>c_{1}^{0}$, the line $c_{1}=t$ intersects these two curves at $\left(t, c_{2}^{*}(t)\right)$ resp. $\left(t, c_{2}^{* *}(t)\right)$, as implied by the very definition of the two quantities $c_{2}^{*}\left(c_{1}\right)$ and $c_{2}^{* *}\left(c_{1}\right)$.

Figure (b) represents some bifurcating solutions corresponding to three values $\alpha, \beta$ and $\gamma$ of the parameter $c_{1}>c_{1}^{0}$. The retained values are here assumed to satisfy $c_{2}^{*}(\alpha)<c_{2}^{* *}(\alpha)$, resp. $c_{2}^{*}(\beta)=c_{2}^{* *}(\beta)$, resp. $c_{2}^{*}(\gamma)>c_{2}^{* *}(\gamma)$. For each $c_{1}>c_{1}^{0}$, there is a coexistence solution joining $\left(R, U^{*}\left(c_{1}\right), 0\right)$ and $\left(R, 0, V^{*}\left(c_{1}\right)\right)$.

are continuous and increasing. Moreover, for $\{i, j\}=\{1,2\}$, we have

$$
\lim _{c_{i} \rightarrow c_{i}^{0}} c_{j}^{*}\left(c_{i}\right)=c_{j}^{0}, \quad \text { and } \lim _{c_{i} \rightarrow+\infty} c_{j}^{*}\left(c_{i}\right)=+\infty .
$$

(iii) With the notation (2.21), whenever $\left(c_{1}, c_{2}\right) \in \Theta$, system 1.2 has a coexistence solution $(R, U, V) \in\left(X_{+}^{*}\right)^{3}$, and we have

$$
\begin{aligned}
& \Theta=\Theta_{-} \cup \Theta_{+}, \text {with } \Theta_{-}=\left\{\left(c_{1}, c_{2}\right), \quad c_{1}<c_{1}^{*}\left(c_{2}\right) \text { and } c_{2}<c_{2}^{*}\left(c_{1}\right)\right\} \text {, } \\
& \text { and } \Theta_{+}=\left\{\left(c_{1}, c_{2}\right), \quad c_{1}>c_{1}^{*}\left(c_{2}\right) \text { and } c_{2}>c_{2}^{*}\left(c_{1}\right)\right\} \text {. }
\end{aligned}
$$

The next sections are devoted to the proof of Theorem 2.11 (trivial solutions), Theorem 2.12 (semi-trivial solutions), as well as Theorems 2.14 and 2.16 (coexistence solutions and coexistence domain).

\section{Zero species: trivial solutions - Proof of Theorem 2.11}

We prove here the various statements of Theorem 2.11. Recall that the problem with zero species reads, shortly, $A_{0} R=I$.

Point (i). Existence and uniqueness of $S$ is clear.

Point (ii). Let $(R, U, V) \in X_{+}^{3}$ be a solution to 1.2 with $U \geq 0$ and $V \geq 0$. We have $A_{0} R=I-c_{1} f_{1}(x, R) U-c_{2} f_{2}(x, R) V \leq I$. Hence $A_{0} R \leq I$ with $A_{0} R \not \equiv I$ whenever 
$U \not \equiv 0$ or $V \not \equiv 0$. The strong maximum principle provides $0<R<S$, with $R<S$ whenever $U \not \equiv 0$ or $V \not \equiv 0$.

Point (iii). Take $w \in X_{+}^{*}$. Due to Assumption 1 for $\varepsilon>0$ small enough, $S$ resp. $\varepsilon$ are upper resp. lower solutions to

$$
A_{0} R+c_{i} f_{i}(x, R) w=I
$$

As a consequence, there exists a pair $\left(R^{-}, R^{+}\right) \in X^{2}$ of maximal solutions to (3.1), with $0<R^{-} \leq R^{+}<S$. Let us show that $R^{-} \equiv R^{+}$. We have $A_{0}\left(R^{+}-R^{-}\right)+$ $c_{i}\left(f_{i}\left(x, R^{+}\right)-f_{i}\left(x, R^{-}\right)\right) w=0$. Integrating over $\Omega$ and taking the boundary conditions into account 18 we obtain

$$
\int_{\Omega}\left[m_{0}\left(R^{+}-R^{-}\right)+c_{i}\left(f_{i}\left(x, R^{+}\right)-f_{i}\left(x, R^{-}\right)\right) w\right] d x=0 .
$$

Since $R \mapsto f_{i}(x, R)$ is an increasing function of $R$ for any value of $x$, we recover $R^{-}=R^{+}$. Existence and uniqueness of $R_{w}^{(i)}$ in the Theorem follows.

Lastly, take $0<w_{1}<w_{2}$, with $w_{1}, w_{2} \in X$. We have $A_{0} R_{w_{2}}^{(i)}+c_{i} f_{i}\left(x, R_{w_{2}}^{(i)}\right) w_{1} \leq$ $I$. Hence, $R_{w_{2}}^{(i)} \in X$ is a lower-solution to $A_{0} R+c_{i} f_{i}(x, R) w_{1}=I$. As a consequence, there exists an actual solution $\widetilde{R}_{w_{1}}^{(i)} \in X$ to $A_{0} R+c_{i} f_{i}(x, R) w_{1}=I$, which satisfies $R_{w_{2}}^{(i)}<\widetilde{R}_{w_{1}}^{(i)}<S$. Uniqueness then provides $\widetilde{R}_{w_{1}}^{(i)}=R_{w_{1}}^{(i)}$. We recover the necessary relation $R_{w_{2}}^{(i)}<R_{w_{1}}^{(i)}$. This ends the proof.

\section{One species: semi-trivial solutions - Proof of Theorem 2.12}

In this section, we study the one species problem (2.18), corresponding to the semitrivial solution $(R, U, 0) \in X_{+}^{*} \times X_{+}^{*} \times X_{+}$to 1.2 . Recall that the one species problem reads

$$
\left\{\begin{array}{l}
A_{0} R+c_{1} f_{1}(x, R) U=I, \\
A_{1} U-c_{1} f_{1}(x, R) U=0 .
\end{array}\right.
$$

4.1 General facts about the one species problem

Lemma 4.1 Let $c_{1}>0$ be fixed. There exists $M_{0}>0$ such that each solution $(R, U) \in$ $\left(X_{+}^{*}\right)^{2}$ to 2.18 verifies

$$
0 \leq U \leq M_{0}
$$

Proof of Lemma 4.1. Let $(R, U) \in\left(X_{+}^{*}\right)^{2}$ be a solution to (2.18). Summing the equations on $R$ and $U$ provides, as already noted, $A_{0} R+A_{1} U=I$. As a consequence, for some $\alpha>0$ small enough we have

$$
(\alpha-\Delta)\left(a_{0} R+a_{1} U\right) \leq I .
$$

18 Robin boundary conditions would add a term $\int_{\partial \Omega} b_{0}(x)\left[R^{+}-R^{-}\right] \geq 0$, and the conclusion would remain unchanged. 
The strong maximum principle 19 then provides $0 \leq a_{0} R+a_{1} U \leq \frac{1}{\alpha}\|I\|_{L^{\infty}}$. In the case of variable coefficients $a_{i}(x)$ with $a_{i}(x)=\lambda_{i} a_{0}(x)$, see footnote 2 , the argument is the same, due to the bound $\left(\alpha-\operatorname{div} a_{0}(x) \nabla\right)\left(R+\lambda_{1} U\right) \leq I=\left(m_{0}(x)-\operatorname{div} a_{0}(x) \nabla\right) R$ $+\left(m_{1}(x)-\lambda_{1} \operatorname{div} a_{0}(x) \nabla\right) U$.

Lemma 4.2 The eigenvalue problem $A_{1} \phi-\mu f_{1}(x, S) \phi=0$ with $\phi \in X$ has a principal eigenvalue $c_{1}^{0}>0$ and a corresponding eigenfunction $\phi_{0} \in X_{+}^{*}$, unique up to a multiplicative constant. We have

$$
A_{1} \phi_{0}-c_{1}^{0} f_{1}(x, S) \phi_{0}=0
$$

with $c_{1}^{0}$ given by $c_{1}^{0}=\min _{\phi \in H^{1}(\Omega), \phi \neq 0}\left[\int a_{1} \nabla \phi^{2}+m_{1} \phi^{2}\right] /\left[\int f_{1}(x, S) \phi^{2}\right]$.

Proof of Lemma 4.2. This is a direct application of Lemma 2.2

Proposition 4.3 Let $c_{1}>0$ be fixed. Suppose there exists $(R, U) \in\left(X_{+}^{*}\right)^{2}$ solution to 2.18. Then we necessarily have $c_{1}>c_{1}^{0}$.

Proof of Proposition 4.3. The function $U>0$ verifies $A_{1} U-c_{1} f_{1}(R) U=0$. Multiplying by $\phi_{0}$, defined in Lemma 4.2 , and integrating over $\Omega$ leads to

$$
0=\int_{\Omega} A_{1} U \phi_{0}-c_{1} \int_{\Omega} f_{1}(x, R) U \phi_{0}=\int_{\Omega} U \phi_{0}\left(c_{1}^{0} f_{1}(x, S)-c_{1} f_{1}(x, R)\right) .
$$

Since Proposition 2.11 ensures $R<S$ hence $f_{1}(x, R)<f_{1}(x, S)$, we recover the necessary condition $c_{1}>c_{1}^{0}$.

4.2 Existence, uniqueness, and some properties of solutions to the one species problem

The main result of this paragraph is the

Proposition 4.4 Suppose Assumptions 1 and 2 are verified. Assume $c_{1}>c_{1}^{0}$.

Then, system 2.18 has a unique solution in $\left(X_{+}^{*}\right)^{2}$, denoted by $\left(R_{u}^{*}\left(c_{1}\right), U^{*}\left(c_{1}\right)\right)$.

Proof of Proposition 4.4. Take a solution $(R, U) \in\left(X_{+}^{*}\right)^{2}$ to 2.18. Defining, as in 2.9 , the quantity $R_{1}(U) \in X$ by the relation $A_{0} R_{1}(U)+A_{1} U=I$ we recover the necessary condition $R=R_{1}(U)$, and system 2.18 can be rewritten (with $\partial_{n} U=0$ on $\partial \Omega)$

$$
A_{1} U-c_{1} f_{1}\left(x, R_{1}(U)\right) U=0,
$$

Let $\phi_{0}>0$ be the eigenfunction defined in Lemma 4.2 , which satisfies $A_{1} \phi_{0}-$ $c_{1}^{0} f_{1}(x, S) \phi_{0}=0$. We claim that for $\varepsilon>0$ small enough and $M>0$ large enough, the pair $\left(\varepsilon \phi_{0}, M\right)$ is a pair of lower-upper solutions to 4.2. Indeed, on the one hand, choosing $M>0$ large enough leads to $R_{1}(M)<0$ (since $A_{0} R_{1}(M)=I-A_{1} M=$ $\left.I-m_{1}(x) M\right)$. Therefore, we obtain

$$
A_{1} M-c_{1} M f_{1}\left(x, R_{1}(M)\right) \geq m_{1} M \geq 0
$$

\footnotetext{
19 with the obvious adaptation in the case of Robin boundary conditions.
} 
with $\partial_{n} M=0$ on $\partial \Omega$, and $M$ is an upper-solution to 4.2 . On the other hand, taking $\varepsilon>0$ small enough leads to

$$
\begin{gathered}
A_{1}\left(\varepsilon \phi_{0}\right)-c_{1} f_{1}\left(x, R_{1}\left(\varepsilon \phi_{0}\right)\right) \cdot\left(\varepsilon \phi_{0}\right)=\varepsilon \phi_{0}\left(c_{1}^{0} f_{1}(x, S)-c_{1} f_{1}\left(x, R_{1}\left(\varepsilon \phi_{0}\right)\right)\right), \\
\quad \text { with } \quad A_{0} R_{1}\left(\varepsilon \phi_{0}\right)+\varepsilon c_{1}^{0} f_{1}(x, S) \phi_{0}=I .
\end{gathered}
$$

It is clear that $\lim _{\varepsilon \rightarrow 0}\left\|R_{1}\left(\varepsilon \phi_{0}\right)-S\right\|_{\infty}=0$. Therefore, we recover

$$
A_{1}\left(\varepsilon \phi_{0}\right)-c_{1} f_{1}\left(x, R_{1}\left(\varepsilon \phi_{0}\right)\right) \cdot\left(\varepsilon \phi_{0}\right)=\varepsilon\left(c_{1}^{0}-c_{1}\right) \phi_{0} f_{1}(x, S)+o_{\varepsilon \rightarrow 0}(1) \leq 0,
$$

with $\partial_{n}\left(\varepsilon \phi_{0}\right)=0$ on $\partial \Omega$. Therefore $\varepsilon \phi_{0}$ is a lower solution to 4.2 for $\varepsilon$ small enough.

These considerations allow us to conclude (see Theorem 2.7) that there exists a pair $\left(U^{-}, U^{+}\right)$of maximal solutions to 4.2 , satisfying $\varepsilon \phi_{0}<U^{-} \leq U^{+}<M$, and for any solution $U \in\left[\varepsilon \phi_{0}, M\right]$ to 4.2 we necessarily have $U^{-} \leq U \leq U^{+}$. Besides, Lemma 4.1 ensures one can choose $M \geq M_{0}$ such that any solution $U \in X_{+}^{*}$ to 4.2 anyhow satisfies $0 \leq U \leq M$. Remembering that 0 is a lower-solution, we thus obtain that every solution $U \in X_{+}^{*}$ necessarily verifies $0 \leq U \leq U^{+}$as well.

Let us show that $U=U^{+}$. We first observe that the relation $0 \leq U \leq U^{+}$implies

$$
0 \leq R_{1}\left(U^{+}\right) \leq R_{1}(U)
$$

This is due to Theorem 2.11 together with the fact that $R_{1}(U)=R_{U}^{(1)}$ and $R_{1}\left(U^{+}\right)=$ $R_{U^{+}}^{(1)}$ in the present case (for $U$ and $U^{+}$solve the auxiliary equation $A_{1} U=c_{1} f_{1}(\ldots) U$ and similarly for $\left.U^{+}\right)$. We deduce $f_{1}\left(x, R_{1}\left(U^{+}\right)\right) \leq f_{1}\left(x, R_{1}(U)\right)$. On the other hand, the obvious integration by parts, together with the definition of $U$ and $U^{+}$, provide

$$
0=\int_{\Omega}\left(\left[A_{1} U\right] U^{+}-\left[A_{1} U^{+}\right] U\right)=\int_{\Omega} c_{1} U U^{+}\left(f_{1}\left(x, R_{1}(U)\right)-f_{1}\left(x, R_{1}\left(U^{+}\right)\right)\right) .
$$

Therefore we obtain $f_{1}\left(x, R_{1}(U)\right)=f_{1}\left(x, R_{1}\left(U^{+}\right)\right)$, hence $R_{1}(U)=R_{1}\left(U^{+}\right)$. Eventually we deduce, using the equations satisfied by $U$ and $U^{+}$again, the relation $U=U^{+}$.

The same proof works in the case of Robin boundary conditions.

With the above Proposition at hand, we complete the picture by stating some properties of the pair $\left(R_{u}^{*}\left(c_{1}\right), U^{*}\left(c_{1}\right)\right)$. We begin with the asymptotic behaviour as $c_{1} \rightarrow \infty$.

Proposition 4.5 With the notation of Proposition 4.4, we have

$$
\lim _{c_{1} \rightarrow+\infty}\left(\left\|R_{u}^{*}\left(c_{1}\right)\right\|_{\infty}+\left\|U^{*}\left(c_{1}\right)-U_{\infty}\right\|_{\infty}\right)=0
$$

where $U_{\infty}$ is the unique solution to $A_{1} U=I$ in $X_{+}$.

Proof of Proposition 4.5 .

Firstly, the function $U_{\infty}$ is an upper-solution to $A_{1} U-c_{1} f_{1}\left(x, R_{1}(U)\right) U=0$ in $X_{+}^{*}$. Indeed, we clearly have, using the definition of $U_{\infty}$ and $R_{1}(\ldots)$, the relation $R_{1}\left(U_{\infty}\right)=0$, from which it follows that $A_{1} U_{\infty}-c_{1} f_{1}\left(x, R_{1}\left(U_{\infty}\right)\right) U_{\infty}=I \geq 0$. 
On the other hand, take an $\varepsilon>0$ fixed. For $c_{1}$ large enough, the function $(1-\varepsilon) U_{\infty}$ is a lower-solution to $A_{1} U-c_{1} f_{1}\left(x, R_{1}(U)\right) U=0$ in $X_{+}^{*}$. Indeed, we have $R_{1}((1-$ $\left.\varepsilon) U_{\infty}\right)=\varepsilon K_{0}(I)=\varepsilon S>0$ on $\bar{\Omega}$, so that

$$
\begin{gathered}
(1-\varepsilon) A_{1} U_{\infty}-c_{1} f_{1}\left(x, R_{1}\left((1-\varepsilon) U_{\infty}\right)\right)(1-\varepsilon) U_{\infty} \\
=(1-\varepsilon)\left[I-c_{1} f_{1}(x, \varepsilon S) U_{\infty}\right]<0,
\end{gathered}
$$

on $\bar{\Omega}$, whenever $c_{1}$ is chosen large enough.

The maximum principle, as stated in Theorem 2.7. establishes that there is a maximal pair $\left(U^{-}, U^{+}\right)$of solutions to $A_{1} U-c_{1} f_{1}\left(x, R_{1}(U)\right) U=0$, satisfying $0<$ $(1-\varepsilon) U_{\infty} \leq U^{-} \leq U^{+} \leq U_{\infty}$. Lastly, we readily know that $U^{*}\left(c_{1}\right)$ is the unique positive solution of $A_{1} U-c_{1} f_{1}\left(x, R_{1}(U)\right) U=0$ so that $U^{-}=U^{+}=U^{*}\left(c_{1}\right)$.

In particular, we recover $(1-\varepsilon) U_{\infty} \leq U^{*}\left(c_{1}\right) \leq U_{\infty}$. This shows $\lim _{c_{1} \rightarrow+\infty} \| U^{*}\left(c_{1}\right)-$ $U_{\infty} \|_{\infty}=0$.

Next, we observe that $R_{u}^{*}\left(c_{1}\right)$ satisfies $A_{0} R_{u}^{*}\left(c_{1}\right)+A_{1} U^{*}\left(c_{1}\right)=I=A_{1} U_{\infty}$, so that formula 2.9 provides

$$
R_{u}^{*}\left(c_{1}\right)=-\frac{a_{1}}{a_{0}} K_{0} A_{0}\left(U^{*}\left(c_{1}\right)-U_{\infty}\right)+\frac{1}{a_{0}} K_{0}\left[\left(a_{1} m_{0}-a_{0} m_{1}\right)\left(U^{*}\left(c_{1}\right)-U_{\infty}\right)\right] .
$$

(with the similar formula if the coefficients $a_{i}$ become space-dependent, with $a_{1}(x)=$ $\lambda_{1} a_{0}(x)$ and $a_{2}(x)=\lambda_{2} a_{0}(x)$ - see footnotes 2, 4 and 9). Using the fact that $U^{*}\left(c_{1}\right) \leq$ $U_{\infty}$, Assumption 2 and, more precisely, relations 2.10 and 2.11, give $0 \leq R_{u}^{*}\left(c_{1}\right) \leq$ $\frac{a_{1}}{a_{0}}\left(U_{\infty}-U^{*}\left(c_{1}\right)\right)$. Using the established limiting behaviour of $U^{*}\left(c_{1}\right)$ we deduce $\lim _{c_{1} \rightarrow+\infty}\left\|R_{u}^{*}\left(c_{1}\right)\right\|_{\infty}=0$.

The next result is a monotonicity property.

Proposition 4.6 With the notation of Proposition 4.4 the map $c_{1} \mapsto U^{*}\left(c_{1}\right)$ is increasing from $\left(c_{1}^{0},+\infty\right)$ to $X_{+}^{*}$, while the map $c_{1} \mapsto R_{u}^{*}\left(c_{1}\right)$ is decreasing from $\left(c_{1}^{0},+\infty\right)$ to $X_{+}^{*}$.

\section{Proof of Proposition 4.6}

Take $b_{2}>b_{1}>c_{1}^{0}$. For $i=1,2$ the function $U^{*}\left(b_{i}\right)$ is the only solution in $X_{+}$to

$$
A_{1} U^{*}\left(b_{i}\right)-b_{i} f_{1}\left(x, R_{1}\left(U^{*}\left(b_{i}\right)\right)\right) U^{*}\left(b_{i}\right)=0 .
$$

We observe that

$$
A_{1} U^{*}\left(b_{1}\right)-b_{2} f_{1}\left(x, R_{1}\left(U^{*}\left(b_{1}\right)\right)\right) U^{*}\left(b_{1}\right)=\left(b_{1}-b_{2}\right) f_{1}\left(x, R_{1}\left(U^{*}\left(b_{1}\right)\right)\right) U^{*}\left(b_{1}\right)<0,
$$

hence $U^{*}\left(b_{1}\right)$ is a lower-solution to $A_{1} U-b_{2} f_{1}\left(x, R_{1}(U)\right) U=0$ in $X_{+}$. On the other hand, we have already established that $U_{\infty}>U^{*}\left(b_{1}\right)$ is an upper-solution as well. Hence the maximum principle, as stated in Theorem 2.7, allows to conclude that there exists a solution $\widetilde{U}\left(b_{2}\right)$ to $A_{1} U-b_{2} f_{1}\left(x, R_{1}(U)\right) U=0$ in $X$ which satisfies $U\left(b_{1}\right)<$ $\widetilde{U}\left(b_{2}\right) \leq U_{\infty}$. The uniqueness we proved in Proposition 4.4 then provides $\widetilde{U}\left(b_{2}\right)=$ $U\left(b_{2}\right)$. Therefore we have $U^{*}\left(b_{1}\right)<U^{*}\left(b_{2}\right)$.

From this we deduce, using the already observed fact that $R_{1}\left(U^{*}\left(b_{i}\right)\right) \equiv R_{U^{*}\left(b_{i}\right)}^{(1)}$, (by definition of the various objects), and using Theorem 2.11 part (iii), the relation $R_{u}^{*}\left(b_{1}\right)>R_{u}^{*}\left(b_{2}\right)$. This ends the proof. 
4.3 Non-degeneracy and index of the semi-trivial solutions

The previous paragraph, and more precisely Proposition 4.4 shows that two families of solutions to the one-species problem 2.18 coexist whenever $c_{1}>c_{1}^{0}$, namely the trivial $\left(c_{1}, S, 0\right)$ and the semi-trivial $\left(c_{1}, R_{u}^{*}\left(c_{1}\right), U^{*}\left(c_{1}\right)\right)$. As an immediate consequence, it appears that $\left(c_{1}^{0}, S, 0\right) \in \mathbb{R} \times\left(X_{+}\right)^{2}$ is a bifurcation point for system (2.18). Note that the bifurcation solution $\left(c_{1}, R_{u}^{*}\left(c_{1}\right), U^{*}\left(c_{1}\right)\right)$ is readily constructed for all values $c_{1}>$ $c_{1}^{0}$, without using the Crandall-Rabinowitz theorem, so that it is not even clear that the branch $\left(c_{1}, R_{u}^{*}\left(c_{1}\right), U^{*}\left(c_{1}\right)\right)$ actually coincides with a bifurcation in the CrandallRabinowitz sense (for instance, the limit as $c_{1} \rightarrow c_{1}^{0}$ of $\left(R_{u}^{*}\left(c_{1}\right), U^{*}\left(c_{1}\right)\right)$ may well differ from $(S, 0)$ at this stage).

In this section, we show essentially two results. On the one hand we show that the Crandall-Rabinowitz theorem applies, and uniqueness allows to conclude that the already constructed semi-trivial solution $\left(c_{1}, R_{u}^{*}\left(c_{1}\right), U^{*}\left(c_{1}\right)\right)$ coincides with the one obtained by bifurcation. On the other hand, and as a consequence, we deduce various properties such as the non-degeneracy of the semi-trivial branch, or we compute the index of this branch. This part of the analysis prepares for the next section where we construct coexistence solutions to the full 2-species problem.

We begin with the

Proposition 4.7 (Local bifurcations in the one-species problem 2.18)

With the above notation, let $\phi_{0} \in X_{+}^{*}$ and $c_{1}^{0}>0$ be as in Lemma 4.2. Define $\rho_{0}=c_{1}^{0} K_{0}\left(f_{1}(S) \phi_{0}\right) \in X_{+}^{*}$. On the other hand, recall from 2.19) the definition

$$
T_{1}\left(c_{1}, R, U\right)={ }^{t}\left(K_{0}\left(I-c_{1} f_{1}(x, R) U\right), K_{1}\left(c_{1} f_{1}(x, R) U\right)\right) .
$$

Then, the following holds

(i) The point $\left(c_{1}^{0}, S, 0\right)$ is a bifurcation point for $T_{1}$, in that Theorem 2.9 applies.

In particular, there exists $\varepsilon>0$, and a map $\left(c_{1}(s), \widehat{r}(s), \widehat{u}(s)\right) \in C^{0}\left((-\varepsilon, \varepsilon) ; \mathbb{R} \times X^{2}\right)$, with $c_{1}(0)=c_{1}^{0}, \widehat{r}(0)=\widehat{u}(0)=0$, such that the branch ${ }^{20}$

$$
\left\{\left(c_{1}(s), S-s\left(\rho_{0}+\widehat{r}(s)\right), s\left(\phi_{0}+\widehat{u}(s)\right) \in c_{1}^{0} \times\left(X_{+}^{*}\right)^{2} ; 0<s<\varepsilon\right\}\right.
$$

is a familly of positive solutions to 2.18. We set $R(s)=S-s\left(\rho_{0}+\widehat{r}(s)\right)$ and $U(s)=$ $s\left(\phi_{0}+\widehat{u}(s)\right)$.

Moreover, each solution $\left(c_{1}, R, U\right) \in \mathbb{R} \times\left(X_{+}\right)^{2}$ to 2.18 near $\left(c_{1}^{0}, 0,0\right)$ is either the trivial solution $\left(c_{1}, S, 0\right)$, or it coincides with $\left(c_{1}(s), R(s), U(s)\right)$ for some $s \in(-\varepsilon, \varepsilon)$. In particular, for any $c_{1}>c_{1}^{0}$, close to $c_{1}^{0}$, there exists $s>0$, such that

$$
\left(c_{1}, R_{u}^{*}\left(c_{1}\right), U^{*}\left(c_{1}\right)\right)=\left(c_{1}(s), R(s), U(s)\right) .
$$

(ii) If $s>0$ is small enough, we have

$$
i\left(T_{1}\left(c_{1}(s), \cdot\right),(R(s), U(s))\right)=1 .
$$

Hence for $c_{1}>c_{1}^{0}$ close to $c_{1}^{0}$ we hav ${ }^{21} i\left(T_{1}\left(c_{1}, \cdot\right),\left(R_{u}^{*}\left(c_{1}\right), U^{*}\left(c_{1}\right)\right)\right)=1$.

\footnotetext{
20 Only positive values of the parameter $s$ are retained. This is due to the fact that we only keep track of positive solutions to system 2.18

21 Our proof also shows that all eigenvalues of $\operatorname{Id}-D_{(R, U)} T_{1}\left(c_{1}, R_{u}^{*}\left(c_{1}\right), U^{*}\left(c_{1}\right)\right)$ are less than one when $c_{1}>c_{1}^{0}$ is close to $c_{1}^{0}$, hence the corresponding solution $\left(R_{u}^{*}\left(c_{1}\right), U^{*}\left(c_{1}\right)\right)$ is stable for the time-dependent parabolic problem associated with the present stationary problem.
} 
Remark 4.8 Point (i) establishes that the branch $\left(c_{1}, R_{u}^{*}\left(c_{1}\right), U^{*}\left(c_{1}\right)\right)$ constructed so far coincides at least locally with the bifurcation branch $\left(c_{1}(s), R(s), U(s)\right)$.

Point (ii) plays a crucial rôle later in the analysis, when exhibiting coexistence solutions to the full 2-species system. We stress the fact that the computation of the above index uses tools from bifurcation theory, hence relies on the identification between the bifurcation branch $\left(c_{1}(s), R(s), U(s)\right)$ and the branch $\left(c_{1}, R_{u}^{*}\left(c_{1}\right), U^{*}\left(c_{1}\right)\right)$.

Proof of Proposition 4.7- Point (i).

System 2.18) is equivalent to $T_{1}\left(c_{1}, R, U\right)={ }^{t}(R, U)$, where the (compact, continuous and twice Fréchet differentiable) operator $T_{1}: \mathbb{R} \times X^{2} \rightarrow X^{2} X$, defined in 2.19 , is ${ }^{22} T_{1}\left(c_{1}, R, U\right)={ }^{t}(S, 0)+c_{1}^{t}\left(-K_{0}\left(f_{1}(R) U\right), K_{1}\left(f_{1}(R) U\right)\right)$. We have, for any $c_{1} \in \mathbb{R}$, the relation $T_{1}\left(c_{1}, S, 0\right)={ }^{t}(S, 0)$, which defines the trivial solution to $T_{1}\left(c_{1}, R, U\right)={ }^{t}(R, U)$. Lastly, we define

$$
L_{1}\left(c_{1}\right)=\mathrm{Id}-D_{(R, U)} T_{1}\left(c_{1}, S, 0\right) .
$$

With these notations at hand, we show that the Crandall-Rabinowitz Theorem 2.9 applies at the bifurcation point $\left(c_{1}^{0}, S, 0\right)$.

Firstly, let $(\rho, \phi) \in \operatorname{Ker}\left(L_{1}\left(c_{1}^{0}\right)\right)$. We have

$$
\rho+c_{1}^{0} K_{0}\left(f_{1}(S) \phi\right)=0 \quad \text { and } \phi-c_{1}^{0} K_{1}\left(f_{1}(S) \phi\right)=0 .
$$

If $\phi \equiv 0$, then $\rho \equiv 0$. Hence $\phi \not \equiv 0$ verifies $A_{1} \phi-c_{1}^{0} f_{1}(S) \phi=0$. By Lemma 4.2 we recover, up to a multiplicative constant, the two relations $\phi=\phi_{0}>0$ and $\rho=-c_{1}^{0} K_{0}\left(f_{1}(S) \phi_{0}\right):=-\rho_{0}<0$. This establishes $\operatorname{dim}\left(\operatorname{Ker}\left(L_{1}\left(c_{1}^{0}\right)\right)\right)=1$ and $\operatorname{Ker}\left(L_{1}\left(c_{1}^{0}\right)\right)=\operatorname{span}\left(-\rho_{0}, \phi_{0}\right)$. Next, since $L_{1}\left(c_{1}^{0}\right)$ is a compact perturbation of the identity, its Fredholm index is 0 and $\operatorname{codim}\left(\operatorname{Im}\left(L_{1}\left(c_{1}^{0}\right)\right)\right)=1$. Lastly, there remains to prove the relation $D_{c_{1}} L_{1}\left(c_{1}^{0}\right) \cdot\left(-\rho_{0}, \phi_{0}\right) \notin \operatorname{Im}\left(L_{1}\left(c_{1}^{0}\right)\right)$. A direct computation shows

$$
D_{c_{1}} L_{1}\left(c_{1}^{0}\right) \cdot\left(-\rho_{0}, \phi_{0}\right)={ }^{t}\left(+K_{0}\left(f_{1}(S) \phi_{0}\right),-K_{1}\left(f_{1}(S) \phi_{0}\right)\right) .
$$

Arguing by contradiction, if $D_{c} L_{1}\left(c_{1}^{0}\right) \cdot\left(-\rho_{0}, \phi_{0}\right) \in \operatorname{Im}\left(L_{1}\left(c_{1}^{0}\right)\right)$, there exists $\phi$ and $\rho$ in $X$ such that

$$
\left(+K_{0}\left(f_{1}(S) \phi_{0}\right),-K_{1}\left(f_{1}(S) \phi_{0}\right)\right)=\left(\rho+c_{1}^{0} K_{0}\left(f_{1}(S) \phi\right), \phi-c_{1}^{0} K_{1}\left(f_{1}(S) \phi\right)\right) .
$$

Applying $A_{1}$ to the second equation, multiplying by $\phi_{0}$, integrating ${ }^{23}$ over $\Omega$, and using the fact that $A_{1} \phi_{0}-c_{1}^{0} f_{1}(S) \phi_{0}=0$, leads to $\int_{\Omega} f_{1}(S) \phi_{0}^{2}=0$ so $\phi_{0}=0$, a contradiction.

We have established that Theorem 2.9 may be applied in the present situation, which guarantees the existence of the branch $\left(c_{1}(s), R(s), U(s)\right)$. The uniqueness part of Proposition 4.4 ensures the identification of this branch with $\left(c_{1}, R_{u}^{*}\left(c_{1}\right), U^{*}\left(c_{1}\right)\right)$ whenever $s>0$ and $c_{1}>c_{1}^{0}$.

22 Here and below we abuse notation by writing $f_{1}(R)$ instead of $f_{1}(x, R)$ and so on.

23 Robin boundary conditions lead to the same calculation. 
Proof of Proposition 4.7- Point (ii)

This proof is more delicate and uses more information from local bifurcation theory.

Since the local bifurcation Theorem of Crandall and Rabinowitz applies, it is known (see 4 20, see also [5] p. 179 for more details) that there exists two maps with $C^{1}$ smoothness,

$$
\begin{gathered}
s \mapsto(\mu(s), w(s))=(\mu(s), \rho(s), \phi(s))) \in \mathbb{R} \times X \times X, \\
\text { with } \mu(0)=0, w(0)=\left(-\rho_{0}, \phi_{0}\right), \\
c_{1} \mapsto\left(\gamma\left(c_{1}\right), w_{0}\left(c_{1}\right)\right)=\left(\gamma\left(c_{1}\right), \rho_{0}\left(c_{1}\right), \phi_{0}\left(c_{1}\right)\right) \in \mathbb{R} \times X \times X, \\
\text { with } \gamma\left(c_{1}^{0}\right)=0, w_{0}\left(c_{1}^{0}\right)=\left(-\rho_{0}, \phi_{0}\right)
\end{gathered}
$$

defined in the neighbourhood of $s=0$, resp. $c_{1}=c_{1}^{0}$, such that along the trivial solution $\left(c_{1}, S, 0\right)$, we have

$$
\left[\operatorname{Id}-D_{(R, U)} T_{1}\left(c_{1}, S, 0\right)\right] \cdot w_{0}\left(c_{1}\right)=\gamma\left(c_{1}\right) w_{0}\left(c_{1}\right),
$$

while along the semi-trivial solution $\left(c_{1}(s), R(s), U(s)\right)$ we have

$$
\left[\mathrm{Id}-D_{(R, U)} T_{1}\left(c_{1}(s), R(s), U(s)\right)\right] \cdot w(s)=\mu(s) w(s),
$$

In order to prove that $i\left(T_{1}\left(c_{1}(s), \cdot\right),(R(s), U(s))\right)=1$ for small values of $s>0$, we now show that $D_{(R, U)} T_{1}\left(c_{1}(s), R(s), U(s)\right)$ has no eigenvalue greater than one (see equation (2.15) ), i.e. all eigenvalues of $\operatorname{Id}-D_{(R, U)} T_{1}\left(c_{1}(s), R(s), U(s)\right)$ are positive. Since $\mu(s)$ is the smallest eigenvalue of $\mathrm{Id}-D_{(R, U)} T_{1}\left(c_{1}(s), R(s), U(s)\right.$ ) (thanks to Lemma 2.1. and using the value of the above operator together with the fact that the components of $w(0)=\left(-\rho_{0}, \phi_{0}\right)$ are uniformly negative resp. positive on $\bar{\Omega}$, so that the same property holds for the components of $w(s)=(-\rho(s), \phi(s))$, at least for small values of $s$ ), we therefore need to show $\mu(s)>0$ for small values of $s>0$.

To do so we use the following known fact from local bifurcation theory, (see [5] p. 179), namely

$$
\mu(s)=-s \gamma^{\prime}\left(c_{1}^{0}\right) c_{1}^{\prime}(s)+o(s) \quad \text { as } s \rightarrow 0 .
$$

This is the key piece of information here. There remains to study the signs of the various terms on the right-hand-side of 4.4. Concerning $c_{1}^{\prime}(s)$, if $s>0$ is small enough, we have, by definition of $c_{1}(s)$, the relation

$$
s A_{1}\left(\phi_{0}+\widehat{u}(s)\right)=s c_{1}(s) f_{1}\left(S-s\left(\rho_{0}+\widehat{r}(s)\right)\right)\left(\phi_{0}+\widehat{u}(s)\right) .
$$

Dividing by $s$ and computing $\left.\frac{d}{d s}\right|_{s=0}$, gives

$$
A_{1} \widehat{u}^{\prime}(0)=c_{1}^{0} f_{1}(S) \widehat{u}^{\prime}(0)+c_{1}^{\prime}(0) f_{1}(S) \phi_{0}-c_{1}^{0} D_{R} f_{1}(S) \phi_{0} \rho_{0} .
$$

Multiplying by $\phi_{0}$ and integrating over $\Omega$, then provides, using the fact that $A_{1} \phi_{0}-$ $c_{1}^{0} f_{1}(S) \phi_{0}=0$, the relation $c_{1}^{\prime}(0) \int_{\Omega} f_{1}(S) \phi_{0}^{2}=c_{1}^{0} \int_{\Omega} D_{R} f_{1}(S) \phi_{0}^{2} \rho_{0}$. We recover $c_{1}^{\prime}(0)>$ 0 , hence $c_{1}^{\prime}(s)>0$ for small values $s>0$. Concerning $\gamma^{\prime}\left(c_{1}^{0}\right)$, we start from the relation, valid whenever $c_{1}$ is close to $c_{1}^{0}$,

$$
A_{1} \phi_{0}\left(c_{1}\right)-c_{1} f_{1}(S) \phi_{0}\left(c_{1}\right)=\gamma\left(c_{1}\right) A_{1} \phi_{0}\left(c_{1}\right) .
$$


Applying $\left.\frac{d}{d c_{1}}\right|_{c_{1}=c_{1}^{0}}$, multiplying by $\phi_{0}=\phi_{0}\left(c_{1}^{0}\right)$, using $\gamma\left(c_{1}^{0}\right)=0$, and integrating over $\Omega$ leads th

$$
-\int_{\Omega} f_{1}(S) \phi_{0}^{2}=+\gamma^{\prime}\left(c_{1}^{0}\right) \int_{\Omega} f_{1}(S) \phi_{0}^{2}
$$

Hence $\gamma^{\prime}\left(c_{1}^{0}\right)<0$. Eventually we have established that $\mu(s)>0$ whenever $s>0$ is small. This provides $i\left(T_{1}\left(c_{1}(s), \cdot\right),(R(s), U(s))\right)=1$ whenever $s>0$ is small.

The proof is complete.

The following is an obvious consequence of Theorem 4.7 .

Proposition 4.9 With the notation of Proposition 4.4, we have

$$
\lim _{c_{1} \rightarrow c_{1}^{0}}\left\|R^{*}\left(c_{1}\right)-S\right\|_{\infty}+\left\|U^{*}\left(c_{1}\right)\right\|_{\infty}=0 .
$$

Proof of Proposition 4.9. Using Theorem 4.7, together with the uniqueness statement of Theorem 4.4 we have $(R(s), U(s))=\left(R^{*}\left(c_{1}(s)\right), U^{*}\left(c_{1}(s)\right)\right)$. Since $\lim _{s \rightarrow 0} c_{1}(s)=c_{1}^{0}$, the result follows from the continuity of $s \mapsto(R(s), U(s))$.

The next Proposition is independent from the previous considerations. It states that each semi-trivial solution $\left(R^{*}\left(c_{1}\right), U^{*}\left(c_{1}\right)\right)$ is non-degenerate.

Proposition 4.10 With the notation of Proposition 4.4 for each $c_{1}>c_{1}^{0}$, we have

$$
\operatorname{Ker}\left(\operatorname{Id}-D_{(R, U)} T_{1}\left(c_{1}, R_{u}^{*}\left(c_{1}\right), U^{*}\left(c_{1}\right)\right)\right)=\{0\} .
$$

Proof of Propostion 4.10 .

The proof is by contradiction.

Take $c_{1}>c_{1}^{0}$ and assume 0 is an eigenvalue of $\operatorname{Id}-D_{(R, U)} T_{1}\left(c_{1}, R_{u}^{*}\left(c_{1}\right), U^{*}\left(c_{1}\right)\right)$.

We define, for each $u \in X$, the following auxiliary operator, acting on $X$. Taking a large, fixed number $K>0$, we introduce

$$
u \in X \mapsto H(u):=\left(A_{1}+K\right)^{-1}\left[f_{1}\left(R_{1}(U)\right) U+K U\right] \in X
$$

where $A_{0} R_{1}(u)+A_{1} u=I$ as usual (see 2.9p). Up to the introduction of the terms involving $K$, the function $H$ is essentially the second component of $T_{1}$, evaluated at $\left(R_{1}(U), U\right)$. From the definition of $H$, the following equivalence is clear whenever $U \in$ $X_{+}^{*}$, namely

$$
T_{1}(R, U)={ }^{t}(R, U) \Leftrightarrow\left[R=R_{1}(U) \text { and } H(U)=U .\right]
$$

Hence we readily have $H\left(U^{*}\left(c_{1}\right)\right)=U^{*}\left(c_{1}\right)$, and the equivalence 4.6 also implies, since 0 is an eigenvalue of $\operatorname{Id}-D_{(R, U)} T_{1}\left(c_{1}, R_{u}^{*}\left(c_{1}\right), U^{*}\left(c_{1}\right)\right)$, that 1 is an eigenvalue of $D_{u} H\left(U^{*}\left(c_{1}\right)\right)$ as well.

We claim that the operator $H$ is nondecreasing, i.e. whenever $U$ and $V$ belong to $X$, we have

$$
U \geq V \geq 0 \Longrightarrow H(U) \geq H(V)
$$

This property is actually the reason for our introduction of the parameter $K$. It comes from the fact that, according to Lemma 2.3 from $U \geq V \geq 0$, we deduce $f_{1}\left(R_{1}(U)\right) U-$

\footnotetext{
24 The computation is the same in the case of Robin boundary conditions.
} 
$f_{1}\left(R_{1}(V)\right) V \geq-\gamma(U-V)$ hence $f_{1}\left(R_{1}(U)\right) U-f_{1}\left(R_{1}(V)\right) V+K(U-V) \geq(K-$ $\gamma)(U-V) \geq 0$, and the maximum principle allows to conclude.

Our second claim is

$$
D_{u} H\left(U^{*}\left(c_{1}\right)\right) \cdot U^{*}\left(c_{1}\right)=k U^{*}\left(c_{1}\right), \text { where } k<1 .
$$

(Note that $k$ is a function in $X$ ). This is the key ingredient. It comes from the following computation. We have

$$
\begin{aligned}
D_{u} H & \left(U^{*}\left(c_{1}\right)\right) \cdot U^{*}\left(c_{1}\right)=\left.\frac{d}{d t}\right|_{t=0} H\left((1+t) U^{*}\left(c_{1}\right)\right) \\
= & \left.\frac{d}{d t}\right|_{t=0}\left(A_{1}+K\right)^{-1}\left[f_{1}\left(R_{1}\left((1+t) U^{*}\left(c_{1}\right)\right)\right)+K\right](1+t) U^{*}\left(c_{1}\right) \\
= & \left(A_{1}+K\right)^{-1}\left[f_{1}\left(R_{1}\left(U^{*}\left(c_{1}\right)\right)\right)+K\right] U^{*}\left(c_{1}\right) \\
& +\left(A_{1}+K\right)^{-1}\left[\left.D_{R} f_{1}\left(R_{1}\left(U^{*}\left(c_{1}\right)\right)\right) U^{*}\left(c_{1}\right) \frac{d}{d t}\right|_{t=0} R_{1}\left((1+t) U^{*}\left(c_{1}\right)\right)\right] .
\end{aligned}
$$

On the other hand, we have

$$
\left(A_{1}+K\right)^{-1}\left[f_{1}\left(R_{1}\left(U^{*}\left(c_{1}\right)\right)\right)+K\right] U^{*}\left(c_{1}\right)=H\left(U^{*}\left(c_{1}\right)\right),
$$

while

$$
\begin{aligned}
&\left.\frac{d}{d t}\right|_{t=0} R_{1}\left((1+t) U^{*}\left(c_{1}\right)\right)=\left.\frac{d}{d t}\right|_{t=0} K_{0}\left[I-(1+t) A_{1} U^{*}\left(c_{1}\right)\right] \\
&=-\left.\frac{d}{d t}\right|_{t=0}(1+t) K_{0}\left[c_{1} f_{1}\left(R_{1}\left(U^{*}\left(c_{1}\right)\right)\right) U^{*}\left(c_{1}\right)\right] \\
&=-K_{0}\left[c_{1} f_{1}\left(R_{1}\left(U^{*}\left(c_{1}\right)\right)\right) U^{*}\left(c_{1}\right)\right]=R_{1}\left(U^{*}\left(c_{1}\right)\right)-S<0 .
\end{aligned}
$$

Eventually we have established

$$
D_{u} H\left(U^{*}\left(c_{1}\right)\right) \cdot U^{*}\left(c_{1}\right)=U^{*}\left(c_{1}\right)+\left(R_{1}\left(U^{*}\left(c_{1}\right)\right)-S\right)=: k U^{*}\left(c_{1}\right),
$$

with $k<1$ as claimed. This proves relation 4.8 .

Our third claim is a consequence of the previous one. It somehow asserts that the two functions $(1+\varepsilon) U^{*}\left(c_{1}\right)$ and $(1-\varepsilon) U^{*}\left(c_{1}\right)$ are lower-upper solutions to $H(u)=u$ in a strong sense. Namely, taking a (fixed) parameter $\mu>0$ such that

$$
k+\mu<1 .
$$

We define

$$
H_{\mu}(u):=H(u)+\mu\left(u-U^{*}\left(c_{1}\right)\right) .
$$

We claim that whenever $\varepsilon>0$ is small enough, we have

$$
H_{\mu}\left((1-\varepsilon) U^{*}\left(c_{1}\right)\right) \geq(1-\varepsilon) U^{*}\left(c_{1}\right), H_{\mu}\left((1+\varepsilon) U^{*}\left(c_{1}\right)\right) \leq(1+\varepsilon) U^{*}\left(c_{1}\right) .
$$

This comes from the following expansion

$$
\begin{aligned}
& H_{\mu}\left((1+\varepsilon) U^{*}\left(c_{1}\right)\right)=H_{\mu}\left(U^{*}\left(c_{1}\right)\right)+\varepsilon D_{u} H_{\mu}\left(U^{*}\left(c_{1}\right)\right) \cdot U^{*}\left(c_{1}\right)+\mathcal{O}\left(\varepsilon^{2}\right) \\
& \quad=U^{*}\left(c_{1}\right)+\varepsilon(k+\mu) U^{*}\left(c_{1}\right)+\mathcal{O}\left(\varepsilon^{2}\right) \\
& \quad \leq(1+\varepsilon) U^{*}\left(c_{1}\right),
\end{aligned}
$$


provided $\varepsilon$ is small enough. We have used relation 4.8 together with the fact that $U^{*}\left(c_{1}\right)>0$.

Gathering all the above claims, let us now show that $D_{u} H\left(U^{*}\left(c_{1}\right)\right)$ cannot have 1 as an eigenvalue. Take $\phi \in X(\phi \not \equiv 0)$ such that

$$
D_{u} H\left(U^{*}\left(c_{1}\right)\right) \cdot \phi=\phi .
$$

Up to rescaling $\phi$, we may assume that

$$
-U^{*}\left(c_{1}\right) \leq \phi \leq U^{*}\left(c_{1}\right)
$$

For technical reasons that become clear later, we may rescale $\phi$ again, so as to ensure that there is a point $x_{0} \in \Omega$ such that

$$
(1+\mu) \phi\left(x_{0}\right)>U^{*}\left(c_{1}\right)\left(x_{0}\right),
$$

where $\mu>0$ is as before. The idea is to compute $H_{\mu}\left(U^{*}\left(c_{1}\right)+\varepsilon \phi\right)$ in two different ways, to obtain the desired contradiction.

On the one hand we have, from the relation $(1-\varepsilon) U^{*}\left(c_{1}\right) \leq U^{*}\left(c_{1}\right)+\varepsilon \phi \leq$ $(1+\varepsilon) U^{*}\left(c_{1}\right)$, and using 4.10 , the bounds

$$
H_{\mu}\left(U^{*}\left(c_{1}\right)+\varepsilon \phi\right) \leq H_{\mu}\left((1+\varepsilon) U^{*}\left(c_{1}\right)\right) \leq(1+\varepsilon) U^{*}\left(c_{1}\right),
$$

as well as $H_{\mu}\left(U^{*}\left(c_{1}\right)+\varepsilon \phi\right) \geq H_{\mu}\left((1-\varepsilon) U^{*}\left(c_{1}\right)\right) \geq(1-\varepsilon) U^{*}\left(c_{1}\right)$. On the other hand, we may expand (the expansion holds in $X$ )

$$
\begin{aligned}
H_{\mu}\left(U^{*}\left(c_{1}\right)+\varepsilon \phi\right)=H_{\mu}\left(U^{*}\left(c_{1}\right)\right)+\varepsilon D_{u} H_{\mu}\left(U^{*}\left(c_{1}\right)\right) \cdot \phi+\mathcal{O}\left(\varepsilon^{2}\right) \\
\quad=U^{*}\left(c_{1}\right)+(1+\mu) \varepsilon \phi+\mathcal{O}\left(\varepsilon^{2}\right) \\
\quad=(1+\varepsilon) U^{*}\left(c_{1}\right)+\varepsilon\left((1+\mu) \phi-U^{*}\left(c_{1}\right)\right)+\mathcal{O}\left(\varepsilon^{2}\right) .
\end{aligned}
$$

Hence, at the point $x_{0}$, we have $H_{\mu}\left(U^{*}\left(c_{1}\right)+\varepsilon \phi\right)\left(x_{0}\right)>(1+\varepsilon) U^{*}\left(c_{1}\right)(0)$, provided $\varepsilon$ is small enough, which contradicts the fact that $H_{\mu}\left(U^{*}\left(c_{1}\right)+\varepsilon \phi\right) \leq(1+\varepsilon) U^{*}\left(c_{1}\right)$.

To summarize, the whole idea of our contradiction argument is that on the one hand $(1+\varepsilon) U^{*}\left(c_{1}\right)$ satisfies $H(U)<U$ in a strict fashion (as a consequence of 4.8 ), while the upper-lower solution technique, together with the fact that $\phi$ is associated with the eigenvalue 1 of the linear part of $H$, imply that when perturbing $U^{*}\left(c_{1}\right)$ in the direction $\phi$, the function $H$ must at the same time be almost constant in that direction and it should decay in a strict fashion as well.

As an immediate consequence of the non-degeneracy of the solution $\left(R_{u}^{*}\left(c_{1}\right), U^{*}\left(c_{1}\right)\right)$, together with the implicit function theorem, we deduce the

Proposition 4.11 The map $c_{1} \mapsto\left(R_{u}^{*}\left(c_{1}\right), U^{*}\left(c_{1}\right)\right)$ is continuously differentiable from $\left(c_{1}^{0},+\infty\right)$ to $X_{+}^{*} \times X_{+}^{*}$.

Proof of Proposition 4.11 .

The pair $\left(R_{u}^{*}\left(c_{1}\right), U^{*}\left(c_{1}\right)\right)$ is defined by the equation

$$
T_{1}\left(c_{1}, R_{u}^{*}\left(c_{1}\right), U^{*}\left(c_{1}\right)\right)={ }^{t}\left(R_{u}^{*}\left(c_{1}\right), U^{*}\left(c_{1}\right) .\right.
$$

On the other hand, we have just proved that $\mathrm{Id}-D_{(R, U)} T_{1}\left(c_{1}, R_{u}^{*}\left(c_{1}\right), U^{*}\left(c_{1}\right)\right)$ does not admit 0 as an eigenvalue, while it is clear from the definition of $T_{1}$ that the linearized operator $D_{(R, U)} T_{1}\left(c_{1}, R, U\right)$ is compact for any value of $\left(c_{1}, R, U\right) \in \mathbb{R} \times X^{2}$. As a consequence, we have that Id $-D_{(R, U)} T_{1}\left(c_{1}, R_{u}^{*}\left(c_{1}\right), U^{*}\left(c_{1}\right)\right)$ is invertible, and the local inversion Theorem applies. 
A key consequence is the following

Proposition 4.12 For any $c_{1}>c_{1}^{0}$, we have

$$
i\left(T_{1}\left(c_{1}, \cdot\right),\left(R_{u}^{*}\left(c_{1}\right), U^{*}\left(c_{1}\right)\right)\right)=1 .
$$

Proof of Proposition 4.12.

Since Id $-D_{(R, U)} T_{1}\left(c_{1}, R_{u}^{*}\left(c_{1}\right), U^{*}\left(c_{1}\right)\right)$ is invertible, and a compact perturbation of the identity, we have

$$
i\left(T_{1}\left(c_{1}, \cdot\right),\left(R_{u}^{*}\left(c_{1}\right), U^{*}\left(c_{1}\right)\right)\right)=(-1)^{p\left(c_{1}\right)},
$$

where $p\left(c_{1}\right)$, is the number of eigenvalues of $D_{(R, U)} T_{1}\left(c_{1}, R_{u}^{*}\left(c_{1}\right), U^{*}\left(c_{1}\right)\right)$ that are greater than 1. Now, take any $c_{1}^{+}>c_{1}^{-}>c_{1}^{0}$. By uniqueness of the solution to $T_{1}\left(c_{1}, R, U\right)=(R, U)$ in $\left(X_{+}^{*}\right)^{2}$, for any $c_{1}>c_{1}^{0}$, we can choose a neighbourhood $\mathcal{U}$ of the set $\left\{\left(c_{1}, R_{u}^{*}\left(c_{1}\right), U^{*}\left(c_{1}\right)\right) ; c_{1} \in\left(c_{1}^{-}, c_{1}^{+}\right)\right\}$in $\mathbb{R} \times\left(X_{+}^{*}\right)^{2}$ such that, if $c_{1} \in\left(c_{1}^{-}, c_{1}^{+}\right)$, no solution solution to $T_{1}\left(c_{1}, R, U\right)=(R, U)$ exists on $\partial \mathcal{U}$. The homotopy conservation (see e.g. 4]) shows that $i\left(T_{1}\left(c_{1}, \cdot\right),\left(R_{u}^{*}\left(c_{1}\right), U^{*}\left(c_{1}\right)\right)\right)$ is constant on $\left(c_{1}^{-}, c_{1}^{+}\right)$. This argument is valid for each $c_{1}^{+}>c_{1}^{-}>c_{1}^{0}$ hence $i\left(T_{1}\left(c_{1}, \cdot\right),\left(R^{*}\left(c_{1}\right), U^{*}\left(c_{1}\right)\right)\right)$ is constant on $\left(c_{1}^{0},+\infty\right)$. We conclude using Proposition 4.7. part (ii), according to which $i\left(T_{1}\left(c_{1}, \cdot\right),\left(R_{u}^{*}\left(c_{1}\right), U^{*}\left(c_{1}\right)\right)\right)=1$ whenever $c_{1}>c_{1}^{0}$ is close to $c_{1}^{0}$. This ends the proof.

\section{Coexistence solutions}

We now show the main result of this paper, namely we exhibit coexistence solutions to the full 2-species system $(1.2)$, i.e. solutions $(R, U, V)$ to 1.2 that lie in $\left(X_{+}^{*}\right)^{3}$. Recall that the system with 2 species reads, shortly,

$$
\left\{\begin{array}{l}
A_{0} R+c_{1} f_{1}(x, R) U+c_{2} f_{2}(x, R) V=I, \\
A_{1} U-c_{1} f_{1}(x, R) U=0 \\
A_{2} V-c_{2} f_{2}(x, R) V=0
\end{array}\right.
$$

\subsection{Preliminary results}

The following fact summarizes the work we have performed at this stage.

Proposition 5.1 The system (1.2) has the trivial solution $(S, 0,0) \in X_{+}^{3}$. Besides,

(i) if $c_{1}>c_{1}^{0}$, system 1.2 has the semi-trivial solution $\left(R_{u}^{*}\left(c_{1}\right), U^{*}\left(c_{1}\right), 0\right) \in X_{+}^{3}$.

(ii) if $c_{2}>c_{2}^{0}$, system 1.2 has the semi-trivial solution $\left(R_{v}^{*}\left(c_{2}\right), 0, V^{*}\left(c_{2}\right)\right) \in X_{+}^{3}$.

We denote these two families by

$$
\begin{aligned}
& \mathscr{C}_{u}=\left\{\left(c_{1}, c_{2}, R_{u}^{*}\left(c_{1}\right), U^{*}\left(c_{1}\right), 0\right),\left(c_{1}, c_{2}\right) \in\left(c_{1}^{0},+\infty\right) \times\left(c_{2}^{0},+\infty\right)\right\}, \\
& \mathscr{C}_{v}=\left\{\left(c_{1}, c_{2}, R_{v}^{*}\left(c_{2}\right), 0, V^{*}\left(c_{2}\right)\right),\left(c_{1}, c_{2}\right) \in\left(c_{1}^{0},+\infty\right) \times\left(c_{2}^{0},+\infty\right)\right\} .
\end{aligned}
$$

Our first result in the direction of obtaining coexistence solutions to (5.1) is the 
Proposition 5.2 Let $\left(c_{1}, c_{2}\right) \in \mathbb{R}^{2}$. Assume that $(R, U, V) \in\left(X_{+}^{*}\right)^{3}$ is a coexistence solution to 5.1.

Then, the following holds:

(i) We necessarily have $c_{1}>c_{1}^{0}$ and $c_{2}>c_{2}^{0}$.

(ii) With the above notation, the function $R-R_{u}^{*}\left(c_{1}\right)$ (resp. $R-R_{v}^{*}\left(c_{2}\right)$ ) either changes sign on $\Omega$, or it vanishes identically.

(iii) We have $0<U<U^{*}\left(c_{1}\right)$ and $0<V<V^{*}\left(c_{2}\right)$ (on $\left.\bar{\Omega}\right)$.

Proof of Proposition 5.2

Let $(R, U, V) \in\left(X_{+}^{*}\right)^{3}$ be a coexistence solution to 1.2 .

Point (i).

By Theorem 2.11 we have $R<S$. Hence, as in the proof of Proposition 4.3, we deduce that $c_{i}>c_{i}^{0}$ for $i=1,2$.

Point (ii).

We have $A_{1} U-c_{1} f_{1}(R) U=A_{1} U^{*}\left(c_{1}\right)-c_{1} f_{1}\left(R_{u}^{*}\left(c_{1}\right)\right) U^{*}\left(c_{1}\right)=0$ with $U>0$ and $U^{*}\left(c_{1}\right)>0$. Hence, by Lemma 2.1 , we recover

$$
\lambda_{1}\left(A_{1}-c_{1} f_{1}(R)\right)=\lambda_{1}\left(A_{1}-c_{1} f_{1}\left(R_{u}^{*}\left(c_{1}\right)\right)\right)=0 .
$$

Point (ii) therefore comes as a direct consequence of the fact that $R \mapsto f_{1}(R)$ increases with $R$, from which it is deduced that $R \mapsto \lambda_{1}\left(A_{1}-c_{1} f_{1}(R)\right)$ decreases with $R$ (Lemma 2.1). The function $R-R_{u}^{*}\left(c_{1}\right)$ cannot have constant sign on $\Omega$, unless it vanishes identically.

Point (iii).

We use a lower-upper solution method. Whenever $u$ and $v$ belong to $X$, denote by $R(u, v)$ the only solution in $X$ to $A_{0} R+A_{1} u+A_{2} v=I$. With this notation at hand, the function $u=U$ is seen to satisfy the following, nonlinear, nonlocal, elliptic problem

$$
A_{1} u-c_{1} f_{1}(R(u, V)) u=0 .
$$

We first claim that $U$ is the only positive solution to 5.2 . To prove this, we observe that whenever $M>0$ is large enough, the constant function $u=M$ is an upper-solution to $(5.2)$. Indeed, it is clear that $R(M, V) \geq 0$ when $M$ is large (for $A_{0}(R(M, V)) \leq 0$ under these circumstances), from which it follows $A_{1} M-c_{1}\left(f_{1}(R(M, V)) M \geq m_{1} M \geq\right.$ 0 . The constant function $u=0$ being clearly a lower-solution to $\sqrt{5.2}$, , it follows that there exist a maximal solution $0 \leq U^{+} \leq M$ such that any solution $u$ to 5.2 such that $0 \leq u \leq M$ also satisfies $0 \leq u \leq U^{+}$. In particular, taking $M>U$, we deduce $0 \leq U \leq U^{+}$.

To prove that $U=U^{+}$, we define for convenience $R^{+}=R\left(U^{+}, V\right)$ and $R=$ $R(U, V)$. We clearly hav£ 25

$$
0=\int_{\Omega}\left(A_{1} U^{+} \cdot U-A_{1} U \cdot U^{+}\right)=c_{1} \int_{\Omega}\left[f_{1}\left(R^{+}\right)-f_{1}(R)\right] U^{+} U,
$$

which proves $U^{+}=U$ provided we establish $R^{+} \leq R$. On the other hand, the function $r=R$ satisfies

$$
A_{0} r+c_{1} f_{1}(r) U=I-c_{2} f_{2}(r) V,
$$

\footnotetext{
25 with the obvious adaptation in the case of Robin boundary conditions
} 
while the function $r=R^{+}$satisfies

$$
A_{0} r+c_{1} f_{1}(r) U^{+}=I-c_{2} f_{2}(r) V
$$

Since $U \leq U^{+}$, we see that $R^{+}$is a lower-solution to 5.3 . This implies, similarly to the proof of the Theorem 2.11 that $R^{+} \leq R$. Hence $U^{+}=U$ and $U$ is the only positive solution to 5.2 .

Let $s \in(0,1)$, we now claim $U^{*}\left(c_{1}\right)$ resp. $s U$ are (strict) upper resp. lower solutions to 5.2 . Indeed, on the one hand, we have

$$
A_{0}\left(R\left(U^{*}\left(c_{1}\right), V\right)-R\left(U^{*}\left(c_{1}\right), 0\right)\right)=-A_{2} V=-c_{2} f_{2}(R(U, V))<0,
$$

so that $R\left(U^{*}\left(c_{1}\right), V\right)<R\left(U^{*}\left(c_{1}\right), 0\right)$. We deduce

$$
\begin{aligned}
& A_{1} U^{*}\left(c_{1}\right)-c_{1} f_{1}\left(R\left(U^{*}\left(c_{1}\right), V\right)\right) U^{*}\left(c_{1}\right) \\
& \quad=c_{1}\left[f_{1}\left(R\left(U^{*}\left(c_{1}\right), 0\right)\right)-f_{1}\left(R\left(U^{*}\left(c_{1}\right), V\right)\right)\right] U^{*}\left(c_{1}\right)>0 .
\end{aligned}
$$

On the other hand, we have

$$
A_{0}(R(s U, V)-R(U, V))=(1-s) c_{1} f_{1}(R(U, V))>0,
$$

so that $R(s U, V)>R(U, V)$. We deduce

$$
A_{1}(s U)-c_{1} f_{1}(R(s U, V)) s U=c_{1}\left[f_{1}(R(U, V))-f_{1}(R(s U, V))\right] s U<0 .
$$

Now, since $\inf \bar{\Omega} U^{*}\left(c_{1}\right)>0$, one can choose $s \in(0,1)$ small enough such that $s U<$ $U^{*}\left(c_{1}\right)$ and it follows that there exists a solution $\widetilde{U}$ to $\sqrt[5.2]{ }$ such that $s U<\widetilde{U}<U^{*}\left(c_{1}\right)$ (the inequalities being strict because $s U$ and $U^{*}\left(c_{1}\right)$ are not true solution). Uniqueness of the positive solution yields $\widetilde{U}=U$ hence $U<U^{*}\left(c_{1}\right)$.

The same proof shows that $V<V^{*}\left(c_{2}\right)$.

To conclude this section, we also state the following two Lemmas.

Lemma 5.3 Let $c_{1}>c_{1}^{0}$.

Then the eigenvalue problem $A_{2} \psi-\mu f_{2}\left(R_{u}^{*}\left(c_{1}\right)\right) \psi=0$ has a principal eigenvalue $c_{2}^{*}\left(c_{1}\right)>0$ and a corresponding eigenfunction $\psi^{*}\left(c_{1}\right)>0$. We have $c_{2}^{*}\left(c_{1}\right)=$ $\min _{\phi \in H^{1}(\Omega), \phi \neq 0} \frac{\int_{\Omega} a_{2} \nabla \phi^{2}+m_{2} \phi^{2}}{\int_{\Omega} f_{2}\left(R_{u}^{*}\left(c_{1}\right)\right) \phi^{2}}$. In particular, there holds $c_{2}^{*}\left(c_{1}\right)>c_{2}^{0}$.

Proof of Lemma 5.3 .

We only need to prove the inequality $c_{2}^{*}\left(c_{1}\right)>c_{2}^{0}$, which comes from the formulae giving $c_{2}^{*}\left(c_{1}\right)$ resp. $c_{2}^{0}$, in conjunction with the maximum principle.

Lemma 5.4 Let $c_{1}>c_{1}^{0}$ be fixed.

Then, there exists $c_{2}^{\max }=c_{2}^{\max }\left(c_{1}\right)>c_{2}^{0}$ such that, if $(R, U, V) \in\left(X_{+}^{*}\right)^{3}$ is a solution of 1.2, we necessarily have $c_{2}<c_{2}^{\max }$.

Proof of Lemma 5.4 Let $c_{1}>c_{1}^{0}$ be given fixed. We suppose by contradiction that there exists a sequence of solutions $\left(c_{2}^{k}, R_{k}, U_{k}, V_{k}\right) \in\left(c_{2}^{0},+\infty\right) \times\left(X_{+}^{*}\right)^{3}$ with $c_{2}^{k} \rightarrow+\infty$.

As in the proof of Lemma 4.1 from the relation $A_{0} R_{k}+A_{1} U_{k}+A_{2} V_{k}=I$ we deduce that for some $\alpha>0$ we have $(\alpha-\Delta)\left(a_{0} R_{k}+a_{1} U_{k}+a_{2} V_{k}\right) \leq I$ (with the obvious adaptation in the case of variable coefficients $a_{i}=a_{i}(x)$, see the proof of Lemma 4.1), hence $0 \leq a_{0} R_{k}+a_{1} U_{k}+a_{2} V_{k} \leq M$ for some $M \geq 0$ independent of $k$. 
We deduce that all functions $R_{k}, U_{k}$, and $V_{k}$ are bounded in $L^{\infty}$, uniformly in $k$. In turn we recover that $A_{0} R_{k}, A_{1} U_{k}$, and $A_{2} V_{k}$ are uniformly bounded in $L^{\infty}$ as well, and a bootstrap argument shows that $R_{k}, U_{k}$, and $V_{k}$ are uniformly bounded in some $C^{2+\beta}$ space $(\beta>0)$, hence converge towards some $R_{\infty}, U_{\infty}, V_{\infty}$ in $X_{+}$, say.

We claim that $R_{\infty}=0$. Indeed, we define the function $v_{k}:=\frac{V_{k}}{c_{2}^{k}\left\|V_{k}\right\|_{\infty}}$ verifies $A_{2} v_{k}=f_{2}\left(R_{k}\right) v_{k}$. It follows that $v_{k}$ converges in $X_{+}^{*}$ to some nonnegative function $v_{\infty}$ verifiyng $A_{2} v_{\infty}=f_{2}\left(R_{\infty}\right) v_{\infty}$. If $R_{\infty} \neq 0$ then $v_{\infty}>0$ which contradicts the fact that $\left\|v_{k}\right\|_{\infty}=\frac{1}{c_{2}^{k}} \rightarrow 0$. We recover $R_{k} \rightarrow 0$ in $X$.

Now, the fact that $U_{k}>0$ provides $\lambda_{1}\left(A_{1}-c_{1} f_{1}\left(R_{k}\right)\right)=0$. We deduce $0=$ $\lambda_{1}\left(A_{1}-c_{1} f_{1}\left(R_{k}\right)\right) \rightarrow \lambda_{1}\left(A_{1}\right)$ as $k \rightarrow \infty$. The known fact $\lambda_{1}\left(A_{1}\right)>0$ provides the contradiction.

\subsection{Proof of Theorem 2.14}

Let us now come to the construction of coexistence solutions.

For a given value of $c_{1}>c_{1}^{0}$, we introduce the (compact, continuous, twice Fréchet differentiable) operator $T_{2}:\left(c_{2}^{0}, \infty\right) \times X^{3} \rightarrow X^{3}$ as

$$
\begin{aligned}
T_{2}\left(c_{2}, R, U, V\right) & =\left(\begin{array}{c}
K_{0}\left(I-c_{1} f_{1}(R) U-c_{2} f_{2}(R) V\right) \\
c_{1} K_{1}\left(f_{1}(R) U\right) \\
c_{2} K_{2}\left(f_{2}(R) V\right)
\end{array}\right) \\
& =\left(\begin{array}{l}
S \\
0 \\
0
\end{array}\right)+c_{1}\left(\begin{array}{c}
-K_{0}\left(f_{1}(R) U\right) \\
K_{1}\left(f_{1}(R) U\right) \\
0
\end{array}\right)+c_{2}\left(\begin{array}{c}
-K_{0}\left(f_{2}(R) V\right) \\
0 \\
K_{2}\left(f_{2}(R) V\right)
\end{array}\right) .
\end{aligned}
$$

Clearly $\left(c_{2}, R, U, V\right) \in\left(c_{2}^{0}, \infty\right) \times\left(X_{+}^{*}\right)^{3}$ is a coexistence solution if and only if

$$
T_{2}\left(c_{2}, R, U, V\right)={ }^{t}(R, U, V) .
$$

We readily know that the semi-trivial solution $\left(c_{2}, R_{u}^{*}\left(c_{1}\right), U^{*}\left(c_{1}\right), 0\right)$ satisfies

$$
T_{2}\left(c_{2}, R_{u}^{*}\left(c_{1}\right), U^{*}\left(c_{1}\right), 0\right)={ }^{t}\left(R_{u}^{*}\left(c_{1}\right), U^{*}\left(c_{1}\right), 0\right),
$$

for any value of $c_{2}$. We now construct coexistence solutions using bifurcations from the (family of) point(s) $\left(c_{2}^{*}\left(c_{1}\right), R_{u}^{*}\left(c_{1}\right), U^{*}\left(c_{1}\right), 0\right)$, where $c_{2}^{*}\left(c_{1}\right)>c_{2}^{0}$ is provided by Lemma 5.3 .

Proposition 5.5 Take $c_{1}>c_{1}^{0}$. Let $c_{2}^{*}=c_{2}^{*}\left(c_{1}\right)>c_{2}^{0}$ be the eigenvalue defined in Lemma 5.3 and $\psi^{*}=\psi^{*}\left(c_{1}\right) \in X_{+}^{*}$ be the associated eigenfunction.

Then $\left(c_{2}^{*}\left(c_{1}\right), R_{u}^{*}\left(c_{1}\right), U^{*}\left(c_{1}\right), 0\right)$ is a bifurcation point for $T_{2}$, in that the local bifurcation Theorem 2.9 applies.

In particular, there exists $\rho^{*}=\rho^{*}\left(c_{1}\right) \in X$ and $\phi^{*}=\phi^{*}\left(c_{1}\right) \in X$, there exists $\varepsilon>0$, there exists a map $(\widetilde{r}, \widetilde{u}, \widetilde{v}) \in C^{1}\left((-\varepsilon, \varepsilon), X^{3}\right)$ verifying $\widetilde{r}(0)=\widetilde{u}(0)=\widetilde{v}(0)=0$, together with a map $c_{2} \in C^{1}\left((-\varepsilon, \varepsilon), \mathbb{R}^{+}\right)$verifying $c_{2}(0)=c_{2}^{*}\left(c_{1}\right)$, such that the following holds. The branch

$$
\left\{\left(c_{2}(s), \widetilde{R}(s), \widetilde{U}(s), \widetilde{V}(s)\right) ; 0<s<\varepsilon\right\}
$$


is a family of positive solutions to (5.1), where we set

$$
\begin{gathered}
\widetilde{R}(s)=R_{u}^{*}\left(c_{1}\right)+s\left(\rho^{*}\left(c_{1}\right)+\widetilde{r}(s)\right), \quad \widetilde{U}(s)=U^{*}\left(c_{1}\right)+s\left(\phi^{*}\left(c_{1}\right)+\widetilde{u}(s)\right), \\
\widetilde{V}(s)=s\left(\psi^{*}\left(c_{1}\right)+\widetilde{v}(s)\right) .
\end{gathered}
$$

Moreover, any solution $\left(c_{2}, R, U, V\right) \in \mathbb{R} \times X^{3}$ to (5.1) near the bifurcation point $\left(c_{2}^{*}\left(c_{1}\right), R_{u}^{*}\left(c_{1}\right), U^{*}\left(c_{1}\right), 0\right)$ is either the semi-trivial solution $\left(c_{2}, R_{u}^{*}\left(c_{1}\right), U^{*}\left(c_{1}\right), 0\right)$, or it coincides for some $s \in(-\varepsilon, \varepsilon)$ with $\left(c_{2}(s), \widetilde{R}(s), \widetilde{U}(s), \widetilde{V}(s)\right)$.

Proof of Proposition 5.5 .

Recall that the value of $c_{1}>c_{1}^{0}$ is fixed. We set

$$
L_{2}\left(c_{2}\right)=\mathrm{Id}-D_{(R, U, V)} T_{2}\left(c_{2}, R_{u}^{*}\left(c_{1}\right), U^{*}\left(c_{1}\right), 0\right) .
$$

Using again the operator $T_{1}$ of the one species problem, see 2.19), we have, whenever $(\rho, \phi, \psi) \in X^{3}$, the relation

$$
\begin{aligned}
& L_{2}\left(c_{2}^{*}\left(c_{1}\right)\right) \cdot{ }^{t}(\rho, \phi, \psi)={ }^{t}(\rho, \phi, \psi) \\
& -\left(\begin{array}{c}
D_{(R, U)} T_{1}\left(c_{1}, R_{u}^{*}\left(c_{1}\right), U^{*}\left(c_{1}\right)\right) \cdot\left(\begin{array}{c}
\rho \\
\phi
\end{array}\right) \\
0
\end{array}\right)-c_{2}^{*}\left(c_{1}\right)\left(\begin{array}{c}
-K_{0}\left(f_{2}\left(R_{u}^{*}\left(c_{1}\right)\right) \psi\right) \\
0 \\
K_{2}\left(f_{2}\left(R_{u}^{*}\left(c_{1}\right)\right) \psi\right)
\end{array}\right) .
\end{aligned}
$$

Take now $(\rho, \phi, \psi) \in \operatorname{Ker}\left(L_{2}\left(c_{2}^{*}\left(c_{1}\right)\right)\right)$. We have

$$
\begin{aligned}
& \left(\operatorname{Id}-D_{(R, U)} T_{1}\left(c_{1}, R_{u}^{*}\left(c_{1}\right), U^{*}\left(c_{1}\right)\right)\right) \cdot{ }^{t}(\rho, \phi)={ }^{t}\left(c_{2}^{*}\left(c_{1}\right) K_{0}\left(f_{2}\left(R_{u}^{*}\left(c_{1}\right)\right) \psi\right), 0\right), \\
& \psi-c_{2}^{*}\left(c_{1}\right) K_{2}\left(f_{2}\left(R_{u}^{*}\left(c_{1}\right)\right) \psi\right)=0 .
\end{aligned}
$$

Equation 5.8 on $\psi$, and the definition of $c_{2}^{*}\left(c_{1}\right)$, implies that $\psi=\psi^{*}\left(c_{1}\right)>0$ up to a multiplicative constant. Equation (5.7) on $(\rho, \phi)$, together with the already proved invertibility of $\operatorname{Id}-D_{(R, U)} T_{1}\left(c_{1}, R_{u}^{*}\left(c_{1}\right), U^{*}\left(c_{1}\right)\right.$ ) (see Proposition 4.10), then provides $(\rho, \phi)=\left(\rho^{*}\left(c_{1}\right), \phi^{*}\left(c_{1}\right)\right)$, where we have set

$$
\begin{aligned}
& { }^{t}\left(\rho^{*}\left(c_{1}\right), \phi^{*}\left(c_{1}\right)\right):= \\
& \quad\left(\operatorname{Id}-D_{(R, U)} T_{1}\left(c_{1}, R_{u}^{*}\left(c_{1}\right), U^{*}\left(c_{1}\right)\right)\right)^{-1} t\left(c_{2}^{*}\left(c_{1}\right) K_{0}\left(f_{2}\left(R_{u}^{*}\left(c_{1}\right)\right) \psi^{*}\left(c_{1}\right)\right), 0\right) .
\end{aligned}
$$

Hence $\operatorname{Ker}\left(L_{2}\left(c_{2}^{*}\left(c_{1}\right)\right)\right)=\operatorname{span}\left(\rho^{*}\left(c_{1}\right), \phi^{*}\left(c_{1}\right), \psi^{*}\left(c_{1}\right)\right)$ and $\operatorname{dim}\left(\operatorname{Ker}\left(L_{2}\left(c_{2}^{*}\left(c_{1}\right)\right)\right)\right)=1$. The Fredholm alternative also provides $\operatorname{codim}\left(\operatorname{Im}\left(L_{2}\left(c_{2}^{*}\left(c_{1}\right)\right)\right)\right)=1$.

There remains to show that

$$
D_{c_{2}} L_{2}\left(c_{2}^{*}\left(c_{1}\right)\right) \cdot{ }^{t}\left(\rho^{*}\left(c_{1}\right), \phi^{*}\left(c_{1}\right), \psi^{*}\left(c_{1}\right)\right) \notin \operatorname{Im}\left(L_{2}\left(c_{2}^{*}\left(c_{1}\right)\right)\right) .
$$

We clearly have

$$
\begin{aligned}
& D_{c_{2}} L_{2}\left(c_{2}^{*}\left(c_{1}\right)\right) \cdot{ }^{t}\left(\rho^{*}\left(c_{1}\right), \phi^{*}\left(c_{1}\right), \psi^{*}\left(c_{1}\right)\right)= \\
& \quad{ }^{t}\left(-K_{0}\left(f_{2}\left(R_{u}^{*}\left(c_{1}\right)\right) \psi^{*}\left(c_{1}\right)\right), 0,-K_{2}\left(f_{2}\left(R_{u}^{*}\left(c_{1}\right)\right) \psi^{*}\left(c_{1}\right)\right)\right) .
\end{aligned}
$$

If relation 5.10 is false, we can find $\psi_{1}$ such that

$$
-K_{2}\left(f_{2}\left(R_{u}^{*}\left(c_{1}\right)\right) \psi^{*}\left(c_{1}\right)\right)=\psi_{1}-c_{2}^{*}\left(c_{1}\right) K_{2}\left(f_{2}\left(R_{u}^{*}\left(c_{1}\right)\right) \psi_{1}\right) .
$$

As in the proof of Proposition 4.7 , we get $\int f_{2}\left(R_{u}^{*}\left(c_{1}\right)\right)\left(\psi^{*}\left(c_{1}\right)\right)^{2}=0$, which contra$\operatorname{dicts} \psi^{*}\left(c_{1}\right)>0$.

Eventually we have proved that the local bifurcation Theorem 2.9 applies, and the Proposition follows. 
Next, a global argument provides the

Proposition 5.6 Let $c_{1}>c_{1}^{0}$ be fixed. Then, equation (5.1) admits a continuum of nontrivial solutions

$$
\left.\mathscr{C}_{0}=\left\{\left(c_{2}, R, U, V\right)\right)\right\} \subset \mathbb{R} \times X^{2} \times(X \backslash\{0\}),
$$

whose closure $\overline{\mathscr{C}_{0}}$ joins the bifurcation point $\left(c_{2}^{*}\left(c_{1}\right), R_{u}^{*}\left(c_{1}\right), U^{*}\left(c_{1}\right), 0\right)$ either to $\infty$ in $\mathbb{R} \times X^{3}$, or to a point $\left(\widehat{c_{2}}, R_{u}^{*}\left(c_{1}\right), U^{*}\left(c_{1}\right), 0\right)$ in $\mathbb{R} \times X^{3}$, where $\widehat{c_{2}} \neq c_{2}^{*}\left(c_{1}\right)$ is such that $\mathrm{Id}-D_{(R, U, V)} T_{2}\left(\widehat{c_{2}}, R_{u}^{*}\left(c_{1}\right), U^{*}\left(c_{1}\right), 0\right)$ is not invertible.

Proof of Proposition 5.6. We apply the global bifurcation Theorem 2.10 It suffices to show that $i\left(T_{2}\left(c_{2}, \cdot\right),\left(R_{u}^{*}\left(c_{1}\right), U^{*}\left(c_{1}\right), 0\right)\right)$ actually changes sign when crossing the value $c_{2}=c_{2}^{*}\left(c_{1}\right)$.

Let $\mu>1$ be an eigenvalue of $D_{(R, U, V)} T_{2}\left(c_{2}, R_{u}^{*}\left(c_{1}\right), U^{*}\left(c_{1}\right), 0\right)=\operatorname{Id}-L_{2}\left(c_{2}\right)$. There exists $(\rho, \phi, \psi) \not \equiv(0,0,0)$ such that $\left(\mathrm{Id}-L_{2}\left(c_{2}\right)\right)^{t}(\rho, \phi, \psi)=\mu^{t}(\rho, \phi, \psi)$.

If $\psi=0$, we recover, using relation (5.6), that

$$
\left(\mathrm{Id}-D_{(R, U)} T_{1}\left(c_{1}, R_{u}^{*}\left(c_{1}\right), U^{*}\left(c_{1}\right)\right)\right)^{t}(\rho, \phi)=\mu^{t}(\rho, \phi) .
$$

Hence $\mu>1$ is an eigenvalue of $\left(\operatorname{Id}-D_{(R, U)} T_{1}\left(c_{1}, R_{u}^{*}\left(c_{1}\right), U^{*}\left(c_{1}\right)\right)\right)$. We know from Proposition 4.12 that such $\mu$ 's are in even number.

If $\psi \neq 0$, we recover using relation (5.6), that $A_{2} \psi-c_{2} f_{2}\left(R_{u}^{*}\left(c_{1}\right)\right) \psi=(1-\mu) A_{2} \psi$, which means,

$$
A_{2} \psi-\frac{c_{2}}{\mu} f_{2}\left(R_{u}^{*}\left(c_{1}\right)\right) \psi=0
$$

Thanks to Lemma 5.3 it becomes clear that the above problem has no nontrivial solution $\psi \not \equiv 0$ whenever $c_{2} \leq c_{2}^{*}\left(c_{1}\right)$, while it has exactly one nontrivial solution (up to a multiplicative constant), namely $\psi^{*}\left(c_{1}\right)$, whenever $c_{2}>c_{2}^{*}\left(c_{1}\right)$ is close enough to $c_{2}^{*}\left(c_{1}\right)$. This establishes

$$
\begin{array}{ll}
i\left(T_{2}\left(c_{2}, \cdot\right),\left(R_{u}^{*}\left(c_{1}\right), U^{*}\left(c_{1}\right), 0\right)\right)=1, & \text { if } c_{2}<c_{2}^{*}\left(c_{1}\right), \\
i\left(T_{2}\left(c_{2}, \cdot\right),\left(R_{u}^{*}\left(c_{1}\right), U^{*}\left(c_{1}\right), 0\right)\right)=-1, & \text { if } c_{2}>c_{2}^{*}\left(c_{1}\right) .
\end{array}
$$

The theorem 2.10 is proved.

At this stage we have exhibited the continuum of nontrivial solutions $\mathscr{C}_{0} \subset \mathbb{R} \times$ $X^{2} \times(X \backslash\{0\})$. We need to select positive solutions (i.e. coexistence solutions) out of $\mathscr{C}_{0}$.

Close to the bifurcation point $\left(c_{2}^{*}\left(c_{1}\right), R_{u}^{*}\left(c_{1}\right), U^{*}\left(c_{1}\right), 0\right)$, the only solutions that belong to $\left(X_{+}^{*}\right)^{3}$ necessarily belong to the branch $\left\{\left(c_{2}(s), \widetilde{R}(s), \widetilde{U}(s), \widetilde{V}(s)\right) ; 0<s<\varepsilon\right\}$, as stated in Proposition 5.5 To transform this construction into a global one, we now define

$$
\begin{aligned}
& \mathscr{C}_{0}^{+} \text {is the closure of the maximal connected componant of } \\
& \qquad \mathscr{C}_{0} \backslash\left\{\left(c_{2}(s), \widetilde{R}(s), \widetilde{U}(s), \widetilde{V}(s)\right) ;-\varepsilon<s<0\right\} .
\end{aligned}
$$

The question we need to address now is whether $\mathscr{C}_{0}^{+} \backslash\left\{\left(c_{2}^{*}\left(c_{1}\right), R_{u}^{*}\left(c_{1}\right), U^{*}\left(c_{1}\right), 0\right)\right\} \subset$ $\mathbb{R} \times\left(X_{+}^{*}\right)^{3}$. The following proposition states that this set cannot remain in $\mathbb{R} \times\left(X_{+}^{*}\right)^{3}$ globally. 
Proposition 5.7 We have

$$
\mathscr{C}_{0}^{+} \backslash\left\{\left(c_{2}^{*}\left(c_{1}\right), R_{u}^{*}\left(c_{1}\right), U^{*}\left(c_{1}\right), 0\right)\right\} \not \subset \mathbb{R} \times\left(X_{+}^{*}\right)^{3} .
$$

Proof of Proposition 5.7

We argue by contradiction. Assume that $\mathscr{C}_{0}^{+} \backslash\left\{\left(c_{2}^{*}\left(c_{1}\right), R_{u}^{*}\left(c_{1}\right), U^{*}\left(c_{1}\right), 0\right)\right\} \subset \mathbb{R} \times$ $\left(X_{+}^{*}\right)^{3}$. The key point is, according to Rabinowitz [2], the subset $\mathscr{C}_{0}^{+}$of $\mathscr{C}_{0}$ satisfies an alternative similar to the one satisfied by $\mathscr{C}_{0}$, namely, one of the three following situations occur:

(i) The set $\mathscr{C}_{0}^{+}$joins $\left(c_{2}^{*}\left(c_{1}\right), R_{u}^{*}\left(c_{1}\right), U^{*}\left(c_{1}\right), 0\right)$ to $\left(\widehat{c}_{2}, R_{u}^{*}\left(c_{1}\right), U^{*}\left(c_{1}\right), 0\right)$ where Id $D_{(R, U, V)} T_{2}\left(\widehat{c}_{2}, R_{u}^{*}\left(c_{1}\right), U^{*}\left(c_{1}\right), 0\right)$ is not invertible and $\widehat{c}_{2} \neq c_{2}^{*}$.

(ii) The set $\mathscr{C}_{0}^{+}$joins $\left(c_{2}^{*}\left(c_{1}\right), R_{u}^{*}\left(c_{1}\right), U^{*}\left(c_{1}\right), 0\right)$ to $\infty$ in $\mathbb{R} \times X^{3}$.

(iii) There exists $\left(c_{2}, R, U, V\right)$ in $\mathscr{C}_{0}^{+}$, such that, writing

$$
\left(c_{2}, R, U, V\right)=\left(c_{2}, R_{u}^{*}\left(c_{1}\right)+r, U^{*}\left(c_{1}\right)+u, v\right),
$$

with $(r, u, v) \neq(0,0,0)$, the symmetric point $\left.\left(c_{2}, R_{u}^{*}\left(c_{1}\right)\right)-r, U^{*}\left(c_{1}\right)-u,-v\right)$ belongs to $\mathscr{C}_{0}^{+}$as well.

In the present contradiction argument, case (iii) cannot occur, nor can case (i) occur. On top of that, take a point $\left(c_{2}, R, U, V\right) \in \mathscr{C}_{0}^{+}$. Lemma 5.4 asserts that we necessarily have $c_{2}^{0}<c_{2}<c_{2}^{\max }\left(c_{1}\right)$. Hence $c_{2}$ remains in a fixed bounded subset of $\mathbb{R}$. Besides, the proof of Lemma 5.4 also asserts that $(R, U, V)$ necessarily belong to a fixed compact subset of $X^{3}$. Hence situation (ii) cannot occur.

This ends the proof.

The above proposition asserts that $\mathscr{C}_{0}^{+}$necessarily leaves the positive cone. The following Lemma provides information on the points where $\mathscr{C}_{0}^{+}$leaves the positive cone.

Lemma 5.8 Take $c_{1}>c_{1}^{0}$. Let $\left(c_{2}, R, U, V\right) \in \mathbb{R} \times\left(X_{+}\right)^{3}$ be the limit, in $\mathbb{R} \times X^{3}$, of a sequence of positive solutions $\left(c_{2}^{k}, R_{k}, U_{k}, V_{k}\right) \in \mathbb{R} \times\left(X_{+}^{*}\right)^{3}$ to 5.1). Then, we have

$$
\lambda_{1}\left(A_{1}-c_{1} f_{1}(R)\right)=\lambda_{1}\left(A_{2}-c_{2} f_{2}(R)\right)=0 .
$$

Proof of Lemma 5.8

For all $k \geq 0$, the function $\psi_{k}=U_{k}\left\|U_{k}\right\|_{X}^{-1}>0$ verifies $A_{1} \psi_{k}-c_{1} f_{1}\left(R_{k}\right) \psi_{k}=0$. Passing to the strong limit and using elliptic regularization provides a $\psi \geq 0$, limit of the $\psi_{k}$ 's, with $\|\psi\|_{X}=1$ and $A_{1} \psi-c_{1} f_{1}(R) \psi=0$. Hence, Lemma 2.2 provides $\psi>0$ and $\lambda_{1}\left(A_{1}-c_{1} f_{1}(R)\right)=0$. The proof for $\lambda_{1}\left(A_{2}-c_{2} f_{2}(R)\right)$ is similar.

The maximum principle now implies the following proposition.

Proposition 5.9 Take $c_{1}>c_{1}^{0}$.

Then, there exists $c_{2}^{* *}\left(c_{1}\right)>c_{2}^{0}$, such that

$$
\begin{aligned}
& \mathscr{C}_{0}^{+} \text {joins }\left(c_{2}^{*}\left(c_{1}\right), R_{u}^{*}\left(c_{1}\right), U^{*}\left(c_{1}\right), 0\right) \\
& \text { to }\left(c_{2}^{* *}\left(c_{1}\right), R_{v}^{*}\left(c_{2}^{* *}\left(c_{1}\right)\right), 0, V^{*}\left(c_{2}^{* *}\left(c_{1}\right)\right)\right) .
\end{aligned}
$$


Proof of Proposition 5.9

Define for convenience $\dot{\mathscr{C}}_{0}^{+}:=\mathscr{C}_{0}^{+} \backslash\left\{\left(c_{2}^{*}\left(c_{1}\right), R_{u}^{*}\left(c_{1}\right), U^{*}\left(c_{1}\right), 0\right)\right\}$.

In the neighbourhood of $\left(c_{2}^{*}\left(c_{1}\right), R_{u}^{*}\left(c_{1}\right), U^{*}\left(c_{1}\right), 0\right)$, we anyhow have $\dot{\mathscr{C}}_{0}^{+} \subset \mathbb{R} \times$ $\left(X_{+}^{*}\right)^{3}$.

On the other hand, by Proposition 5.7 there exists $\left(\widehat{c_{2}}, \widehat{R}, \widehat{U}, \widehat{V}\right)$ in the set $\dot{\mathscr{C}}_{0}^{+} \cap$ $\left(\mathbb{R} \times \partial\left(X_{+}^{*}\right)^{3}\right)$, which is the limit of a sequence of solutions $\left(c_{2}^{k}, R_{k}, U_{k}, V_{k}\right)$ lying in $\dot{\mathscr{C}}_{0}^{+} \cap\left(\mathbb{R} \times\left(X_{+}^{*}\right)^{3}\right)$. In particular, $(\widehat{R}, \widehat{U}, \widehat{V}) \in\left(X_{+}\right)^{3}$ satisfies (5.1), hence for some $x \in \bar{\Omega}$, we have $\widehat{R}(x) \widehat{U}(x) \widehat{V}(x)=0$.

The maximum principle and the Hopf Lemma then assert that $\widehat{R}$ (resp. $\widehat{U}$, resp. $\widehat{V})$ cannot reach its minimal value 0 in $\bar{\Omega}$ unless it is constant. In the case when $\widehat{R} \equiv 0$, we recover $I=0$, which is impossible. It follows that either $\widehat{U} \equiv 0$ or $\widehat{V} \equiv 0$. If $\widehat{U}=\widehat{V} \equiv 0$, then $\left(\widehat{c_{2}}, \widehat{U}, \widehat{V}, \widehat{R}\right)$ is the trivial solution. By Lemma 5.8 this implies that $c_{1}$ is an eigenvalue of $A_{1} \phi-c_{1} f_{1}(S) \phi=0$, hence that $c_{1}=c_{1}^{0}$. This contradicts $c_{1}>c_{1}^{0}$. Now, suppose $\widehat{V} \equiv 0$ and $\widehat{U}>0$. Uniqueness of the semi-trivial solution provides $\widehat{U}=U^{*}\left(c_{1}\right)$ and $\widehat{R}=R_{u}^{*}\left(c_{1}\right)$. By Lemma 5.8, there exists $\psi>0$ satisfying $A_{2} \psi-\widehat{c_{2}} f_{2}\left(R_{u}^{*}\left(c_{1}\right)\right) \psi=0$. Lemma 5.3 then provides the necessary relation $\widehat{c_{2}}=c_{2}^{*}\left(c_{1}\right)$ which is again a contradiction. Eventually, the only possibility is $\widehat{V}>0, \widehat{R}>0$ and $\widehat{U} \equiv 0$. Hence $\left(c_{1}, \widehat{c_{2}}, \widehat{U}, \widehat{V}, \widehat{R}\right) \in \mathscr{C}_{v}$.

Theorem 2.14 is now a combinaison of Propositions 5.6 and 5.9

\subsection{Coexistence domain : proof of Theorem 2.16}

Theorem 2.14 states that two families of coexistence solutions may be obtained, namely the first one is constructed by freezing $c_{1}>c_{1}^{0}$ and seeing $c_{2}$ as a bifurcation parameter to bifurcate from the semi-trivial $\left(c_{2}^{*}\left(c_{1}\right), R_{u}^{*}\left(c_{1}\right), U^{*}\left(c_{1}\right), 0\right)$ where $c_{2}^{*}\left(c_{1}\right)>c_{2}^{0}$, while the second one is constructed by freezing $c_{2}>c_{2}^{0}$ and seeing $c_{1}$ as a bifurcation parameter to bifurcate from the semi-trivial $\left(c_{1}^{*}\left(c_{2}\right), R_{v}^{*}\left(c_{1}\right), 0, V^{*}\left(c_{2}\right)\right)$ where $c_{1}^{*}\left(c_{2}\right)>c_{1}^{0}$. This construction leads to defining the quantities $c_{2}^{* *}\left(c_{1}\right)>c_{2}^{0}$ and $c_{1}^{* *}\left(c_{2}\right)>c_{1}^{0}$. Note that the three situations $c_{2}^{* *}\left(c_{1}\right)>c_{2}^{*}\left(c_{1}\right), c_{2}^{* *}\left(c_{1}\right)<c_{2}^{*}\left(c_{1}\right), c_{2}^{* *}\left(c_{1}\right)=c_{2}^{*}\left(c_{1}\right)$ may very well occur, and similarly for $c_{1}^{* *}\left(c_{2}\right)$ and $c_{1}^{*}\left(c_{2}\right)$.

Let us now exhibit some properties of the $c_{i}^{*}\left(c_{j}\right)$ 's and $c_{i}^{* *}\left(c_{j}\right)$ 's.

Lemma 5.10 For each $c_{1}>c_{1}^{0}$ and $c_{2}>c_{2}^{0}$, we define

$$
\mu\left(c_{1}, c_{2}\right):=\lambda_{1}\left(A_{1}-c_{1} f_{1}\left(R_{v}^{*}\left(c_{2}\right)\right)\right), \quad \nu\left(c_{1}, c_{2}\right):=\lambda_{1}\left(A_{2}-c_{2} f_{2}\left(R_{u}^{*}\left(c_{1}\right)\right)\right) .
$$

We have the relation (where $\operatorname{sgn}(s)=+1$ if $s>0,=-1$ if $s<0$ and $=0$ if $s=0$ )

$$
\begin{aligned}
& \operatorname{sgn}\left(\mu\left(c_{1}, c_{2}\right)\right)=\operatorname{sgn}\left(c_{1}^{*}\left(c_{2}\right)-c_{1}\right)=-\operatorname{sgn}\left(c_{2}^{* *}\left(c_{1}\right)-c_{2}\right), \\
& \operatorname{sgn}\left(\nu\left(c_{1}, c_{2}\right)\right)=\operatorname{sgn}\left(c_{2}^{*}\left(c_{1}\right)-c_{2}\right)=-\operatorname{sgn}\left(c_{1}^{* *}\left(c_{2}\right)-c_{1}\right) .
\end{aligned}
$$

Proof of Lemma 5.10 .

We show the result for $\mu$. The proof for $\nu$ is similar.

Take $c_{2}>c_{2}^{0}$. The definition of $c_{1}^{*}\left(c_{2}\right)$ readily provides $\mu\left(c_{1}^{*}\left(c_{2}\right), c_{2}\right)=\lambda_{1}\left(A_{1}-\right.$ $\left.c_{1}^{*}\left(c_{2}\right) f_{1}\left(R_{v}^{*}\left(c_{2}\right)\right)\right)=0$. On the other hand, Lemma 2.2 states that the map $c_{1} \mapsto$ $\mu\left(c_{1}, c_{2}\right)$ is increasing. This shows that $\operatorname{sgn}\left(\mu\left(c_{1}, c_{2}\right)\right)=\operatorname{sgn}\left(c_{1}^{*}\left(c_{2}\right)-c_{1}\right)$.

Take $c_{1}>c_{1}^{0}$. The construction of the point $\left(c_{2}^{* *}\left(c_{1}\right), R_{v}^{*}\left(c_{2}^{* *}\left(c_{1}\right)\right), 0, V^{*}\left(c_{2}^{* *}\left(c_{1}\right)\right)\right)$, together with Lemma 5.8 , provide $\mu\left(c_{1}, c_{2}^{* *}\left(c_{1}\right)\right)=\lambda_{1}\left(A_{1}-c_{1} f_{1}\left(R_{v}^{*}\left(c_{2}^{* *}\left(c_{1}\right)\right)\right)\right)=0$. By Theorem 2.12, the map $c_{2} \mapsto R_{v}^{*}\left(c_{2}\right)$ is decreasing, hence by Lemma 2.1. the map $c_{2} \mapsto \mu\left(c_{1}, c_{2}\right)$ is increasing. This shows $\operatorname{sgn}\left(\mu\left(c_{1}, c_{2}\right)\right)=-\operatorname{sgn}\left(c_{2}^{* *}\left(c_{1}\right)-c_{2}\right)$. 
With this Lemma at hand, we may now prove the

Proposition 5.11 Let be $\{i, j\}=\{1,2\}$. For all $c_{j}>c_{j}^{0}$, the scalar $c_{i}^{* *}\left(c_{j}\right)$ is characterized by 26

$$
c_{i}^{*}\left(c_{j}^{* *}\left(c_{i}\right)\right)=c_{i} .
$$

Proof of Proposition 5.11 .

Take $c_{1}>c_{1}^{0}$. Set $d_{2}=c_{2}^{* *}\left(c_{1}\right)$, a quantity that is characterized by the fact that $\lambda_{1}\left(A_{1}-c_{1} f_{1}\left(R_{v}^{*}\left(d_{2}\right)\right)=0\right.$, according to the previous Lemma. Now the quantity $d_{1}:=$ $c_{1}^{*}\left(d_{2}\right)$ is in turn characterized by the relation $\lambda_{1}\left(A_{1}-d_{1} f_{1}\left(R_{v}^{*}\left(d_{2}\right)\right)=0\right.$, and the previous relation shows $d_{1}=c_{1}$. This establishes $c_{1}^{*}\left(c_{2}^{* *}\left(c_{1}\right)\right)=c_{1}$. The proof of the relation $c_{2}^{*}\left(c_{1}^{* *}\left(c_{2}\right)\right)=c_{2}$ is the same.

The following Proposition is another consequence of the above Lemma.

Proposition 5.12 (i) The function $c_{1} \mapsto c_{2}^{*}\left(c_{1}\right)$ is continuous and increasing from $\left(c_{1}^{0},+\infty\right)$ to $\left(c_{2}^{0},+\infty\right)$. The similar statement holds for $c_{2} \mapsto c_{1}^{*}\left(c_{2}\right)$.

(ii) We have $\lim _{c_{1} \rightarrow \infty} c_{2}^{*}\left(c_{1}\right)=+\infty$ and $\lim _{c_{2} \rightarrow \infty} c_{1}^{*}\left(c_{2}\right)=+\infty$.

(iii) We have $\lim _{c_{1} \rightarrow c_{1}^{0}} c_{2}^{*}\left(c_{1}\right)=c_{2}^{0}$ and $\lim _{c_{2} \rightarrow c_{2}^{0}} c_{1}^{*}\left(c_{2}\right)=c_{1}^{0}$.

Proof of Proposition 5.12

We prove only the properties concerning the map $c_{2} \mapsto c_{1}^{*}\left(c_{2}\right)$.

Take $c_{2}>c_{2}^{0}$. We have $\lambda_{1}\left(A_{1}-c_{1}^{*}\left(c_{2}\right) f_{1}\left(R_{v}^{*}\left(c_{2}\right)\right)\right)=0$. On the other hand, Theorem 2.12 asserts that the function $c_{2} \mapsto R_{v}^{*}\left(c_{2}\right)$ is continuous and decreasing. Hence, from Lemma 2.1 we deduce that $c_{1} \mapsto c_{2}^{*}\left(c_{1}\right)$ is continuous and increasing.

Now, we have that $R_{v}^{*}\left(c_{2}\right)$ tends uniformly to 0 when $c_{2} \rightarrow \infty$. If $c_{1}^{*}\left(c_{2}\right)$ remains bounded as $c_{2} \rightarrow \infty$, then $\lambda_{1}\left(A_{1}-c_{1}^{*}\left(c_{2}\right) f_{1}\left(R_{v}^{*}\left(c_{2}\right)\right)\right)=0 \rightarrow \lambda_{1}\left(A_{1}\right)>0$ as $c_{2} \rightarrow \infty$, which is impossible. Therefore, we necessarily have $c_{1}^{*}\left(c_{2}\right) \rightarrow \infty$ as $c_{2} \rightarrow \infty$.

Similarly, as $R_{v}^{*}\left(c_{2}\right)$ tends uniformly to $S$ as $c_{2} \rightarrow c_{2}^{0}$, Lemma 5.3 provides the relation $\lim _{c_{2} \rightarrow c_{2}^{0}} c_{1}^{*}\left(c_{2}\right)=c_{1}^{0}$.

At this level of the analysis, one may define the three open sets

$$
\begin{aligned}
& \left.\Theta_{+}=\left\{c_{1}, c_{2}\right) \in\left(c_{1}^{0},+\infty\right) \times\left(c_{2}^{0},+\infty\right), c_{i}^{*}\left(c_{j}\right)<c_{i}<c_{i}^{* *}\left(c_{j}\right), i \neq j\right\}, \\
& \left.\Theta_{-}=\left\{c_{1}, c_{2}\right) \in\left(c_{1}^{0},+\infty\right) \times\left(c_{2}^{0},+\infty\right), c_{i}^{* *}\left(c_{j}\right)<c_{i}<c_{i}^{*}\left(c_{j}\right), i \neq j\right\} . \\
& \Theta=\Theta_{-} \cup \Theta_{+} .
\end{aligned}
$$

It is clear that whenever $\left(c_{1}, c_{2}\right) \in \Theta$, a coexistence solution may be exhibited to 5.1. Note however that these sets may be void. Note as well that our construction anyhow exhibits coexistence solutions for some values of $\left(c_{1}, c_{2}\right)$, obtained by fixing $c_{1}$ and letting $c_{2}$ vary, say: in that respect the set $\Theta$ may not exhaust all values of $\left(c_{1}, c_{2}\right)$ for which a coexistence solution may be exhibited.

In any circumstance, the above results show that these sets have the simpler value

$$
\begin{aligned}
& \left.\Theta_{+}=\left\{c_{1}, c_{2}\right) \in\left(c_{1}^{0},+\infty\right) \times\left(c_{2}^{0},+\infty\right), c_{1}>c_{2}^{*}\left(c_{1}\right), c_{2}>c_{1}^{*}\left(c_{2}\right)\right\} \\
& \left.\Theta_{-}=\left\{c_{1}, c_{2}\right) \in\left(c_{1}^{0},+\infty\right) \times\left(c_{2}^{0},+\infty\right), c_{1}<c_{2}^{*}\left(c_{1}\right), c_{2}<c_{1}^{*}\left(c_{2}\right)\right\}
\end{aligned}
$$

26 That is, $c_{i}^{* *}=\left(c_{i}^{*}\right)^{-1}$. 
For later convenience we also define

$$
\begin{aligned}
& \left.\widetilde{\Theta_{+}}=\left\{c_{1}, c_{2}\right) \in\left(c_{1}^{0},+\infty\right) \times\left(c_{2}^{0},+\infty\right), c_{i}^{*}\left(c_{j}\right) \leq c_{i} \leq c_{i}^{* *}\left(c_{j}\right), i \neq j\right\}, \\
& \left.\widetilde{\Theta_{-}}=\left\{c_{1}, c_{2}\right) \in\left(c_{1}^{0},+\infty\right) \times\left(c_{2}^{0},+\infty\right), c_{i}^{* *}\left(c_{j}\right) \leq c_{i} \leq c_{i}^{*}\left(c_{j}\right), i \neq j\right\} . \\
& \widetilde{\Theta^{\prime}}=\widetilde{\Theta_{-}} \cup \widetilde{\Theta_{+}} .
\end{aligned}
$$

\section{Interpretations, and ecological aspects}

6.1 A conjecture

\section{Conjecture}

(i) If $\left(c_{1}, c_{2}\right) \notin \widetilde{\Theta}$, then there cannot exist $(R, U, V) \in\left(X_{+}^{*}\right)^{3}$ solution to 5.1).

(ii) We have $\Theta_{-}=\emptyset$, or, in other words, $c_{i}^{*}\left(c_{j}\right) \leq c_{i}^{* *}\left(c_{j}\right)$ whenever $i \neq j$.

This conjecture is motivated by our numerical simulations. It states that the set $\widetilde{\Theta}$ actually characterizes those values of $\left(c_{1}, c_{2}\right)$ for which a coexistence solution may be exhibited. It also states that species $i$ survives if and only if $c_{i} \leq c_{i}^{*}\left(c_{j}\right)$. In other words, species $i$ survives if and only if $\lambda_{1}\left(A_{i}-c_{i} f_{i}\left(R^{*}\left(c_{j}\right)\right) \geq 0\right.$.

\subsection{Two ecological properties}

Lemma 5.3 readily provides the following result.

Proposition 6.1 (dependence of the coexistence solutions on the diffusion rates).

Take $a_{0}$ and $a_{1}$ in $(0,+\infty)$, and consider the system (5.1) as a function of the diffusion rate $a_{2}$.

Then, the map $a_{2} \mapsto c_{2}^{*}\left(c_{1}\right)\left(a_{2}\right)$ is increasing.

Moreover, if $x \mapsto m_{2}(x)-f_{2}\left(x, R_{u}^{*}\left(c_{1}\right)(x)\right)$ is not a constant function, then $a_{2} \mapsto$ $c_{2}^{*}\left(c_{1}\right)\left(a_{2}\right)$ is strictly increasing.

Provided the above conjecture holds, this assertion implies that as the diffusion rate of a given species increases, its ability to survive decreases.

Proposition 6.2 (rôle of the heterogeneity).

(i) If $\left(c_{1}, c_{2}\right) \in \Theta$, we necessarily have that $R_{u}^{*}\left(c_{1}\right)-R_{v}^{*}\left(c_{2}\right)$ is neither positive nor negative.

(ii) If $R_{u}^{*}\left(c_{1}\right)=R_{v}^{*}\left(c_{2}\right)$, then, for all $i, j=1,2, i \neq j$, we have $c_{i}^{*}\left(c_{j}\right)=c_{i}^{* *}\left(c_{j}\right)=c_{i}$ and

$$
\left\{\left(c_{1}, c_{2}, R_{u}^{*},(1-t) U^{*}, t V^{*}, t \in[0,1]\right\} \in\left\{c_{1}, c_{2}\right\} \times X_{+}^{3} .\right.
$$

is a familly of solutions joining $\left(c_{1}, c_{2}, R_{u}^{*}\left(c_{1}\right), U^{*}\left(c_{1}\right), 0\right)$ to $\left(c_{1}, c_{2}, R_{v}^{*}\left(c_{2}\right), 0, V^{*}\left(c_{2}\right)\right)$.

In other words, the coexistence domain $\Theta$ is embedded in the set of the $\left(c_{1}, c_{2}\right)$ such that $R_{u}^{*}\left(c_{1}\right)-R_{v}^{*}\left(c_{2}\right)$ is neither positive nor negative. This point highlights the importance of the spatial heterogeneity in the coexistence process. This point in further discussed in the next subsection. 
Proof of Proposition 6.2

If $\left(c_{1}, c_{2}\right) \in \Theta$, then $\mu\left(c_{1}, c_{2}\right) \nu\left(c_{1}, c_{2}\right)>0$. On the other hand, we know that $\lambda_{1}\left(A_{1}-c_{1} f_{1}\left(R_{u}^{*}\left(c_{1}\right)\right)\right)=0$ and $\lambda_{1}\left(A_{2}-c_{2} f_{2}\left(R_{v}^{*}\left(c_{2}\right)\right)\right)=0$. Hence, if $R_{u}^{*}\left(c_{1}\right) \geq$ $R_{v}^{*}\left(c_{2}\right)$, then $\mu\left(c_{1}, c_{2}\right)=\lambda_{1}\left(A_{1}-c_{1} f_{1}\left(R_{v}^{*}\left(c_{2}\right)\right)\right)>0$. Therefore, $\nu\left(c_{1}, c_{2}\right)=\lambda_{1}\left(A_{2}-\right.$ $\left.c_{2} f_{2}\left(R_{u}^{*}\left(c_{1}\right)\right)\right)>0$ as well. Hence $\lambda_{1}\left(A_{2}-c_{2} f_{2}\left(R_{v}^{*}\left(c_{2}\right)\right)\right)>0$, which is impossible. The same arguments shows that $R_{v}^{*}\left(c_{2}\right) \geq R_{u}^{*}\left(c_{1}\right)$ is impossible.

Now, if $R_{u}^{*}\left(c_{1}\right)=R_{v}^{*}\left(c_{2}\right):=R$, then $\mu\left(c_{1}, c_{2}\right)=\nu\left(c_{1}, c_{2}\right)=0$, hence $c_{i}=c_{i}^{*}\left(c_{j}\right)=$ $c_{i}^{* *}\left(c_{j}\right)$. One gets $A_{0} R+c_{1} f_{1}(R) U=A_{0} R+c_{2} f_{2}(R) V$ thus, for all $t \in[0,1]$, we have $A_{0} R+(1-t) c_{1} f_{1}(R) U^{*}+t c_{1} f_{1}(R) V^{*}=I$. Since $A_{1} U^{*}=c_{1} f_{1}(R) U^{*}$ and $A_{2} V^{*}=$ $c_{2} f_{2}(R) V^{*}$, we see that $\left\{\left(c_{1}, c_{2}, R_{u}^{*},(1-t) U^{*}, t V^{*}, t \in[0,1]\right\}\right.$ is a family of solutions. $\square$

6.3 Two degenerate cases

In the homogeneous case where the functions $I(x), f_{i}(x), m_{i}(x), a_{i}(x)$ do not depend on $x$, and when Neumann boundary conditions are retained, we have that $R_{u}^{*}\left(c_{1}\right)(x)$ and $R_{v}^{*}\left(c_{2}\right)(x)$ are constant functions. Hence, by Proposition 6.2 the coexistence is possible only if $R_{u}^{*}\left(c_{2}\right)=R_{u}^{*}\left(c_{1}\right)$, which induces a degenerate solution. Moreover, the fact that $R_{u}^{*}\left(c_{1}\right)$ and $R_{v}^{*}\left(c_{2}\right)$ decrease imply that meas $\left\{\left(c_{1}, c_{2}\right) \in\left(c_{1}^{0},+\infty\right) \times\left(c_{2}^{0},+\infty\right), R_{u}^{*}\left(c_{2}\right)=\right.$ $\left.R_{u}^{*}\left(c_{1}\right)\right\}=0$. In that degenerate case we have the

Proposition 6.3 (The homogeneous case). Assume the problem is homogeneous, i.e. $I, m_{i}$ and $f_{i}$ do not depend on $x$. Assume Neumann boundary conditions are retained.

Then we have $c_{i}^{0}=\inf \left\{c_{i}>0, f_{i}^{-1}\left(m_{i} / c_{i}\right)\right.$ exists and is smaller than $\left.S:=\frac{I}{m_{0}}\right\}$, and the only semi-trivial solutions are $\left(R_{u}^{*}\left(c_{1}\right), U^{*}\left(c_{1}\right), 0\right)$ and $\left(R_{v}^{*}\left(c_{2}\right), 0, V^{*}\left(c_{2}\right)\right)$, where

$$
\begin{aligned}
& R_{u}^{*}\left(c_{1}\right)=f_{1}^{-1}\left(m_{1} / c_{1}\right), \quad R_{v}^{*}\left(c_{2}\right)=f_{2}^{-1}\left(m_{2} / c_{2}\right), \\
& U^{*}\left(c_{1}\right)=\left(I-m_{1} R_{u}^{*}\left(c_{1}\right)\right) / m_{0}, \quad V^{*}\left(c_{2}\right)=\left(I-m_{2} R_{v}^{*}\left(c_{2}\right)\right) / m_{0} .
\end{aligned}
$$

In particular, either $R_{u}^{*}\left(c_{1}\right)>R_{v}^{*}\left(c_{2}\right)$, or $R_{u}^{*}\left(c_{1}\right)<R_{v}^{*}\left(c_{2}\right)$, or $R_{u}^{*}\left(c_{1}\right)=R_{v}^{*}\left(c_{2}\right)$.

In any circumstance, we have

$$
\begin{aligned}
\Theta & =\emptyset \\
\widetilde{\Theta} & =\left\{\left(c_{1}, c_{2}\right) \in\left(c_{1}^{0},+\infty\right) \times\left(c_{2}^{0},+\infty\right) \text { s.t. } R_{1}^{*}\left(c_{1}\right)=R_{2}^{*}\left(c_{2}\right)<S\right\} \\
& =\left\{\left(c_{1}, c_{2}\right) \in\left(c_{1}^{0},+\infty\right) \times\left(c_{2}^{0},+\infty\right) \text { s.t. } f_{1}^{-1}\left(\frac{m_{1}}{c_{1}}\right)=f_{2}^{-1}\left(\frac{m_{2}}{c_{2}}\right)<S\right\} .
\end{aligned}
$$

Moreover, for all $\left(c_{1}, c_{2}\right) \in \widetilde{\Theta}$, there exists a family of solutions $\left\{\left(R_{1}^{*}\left(c_{1}\right), t U^{*}\left(c_{1}\right),(1-\right.\right.$ $\left.\left.t) V^{*}\left(c_{2}\right)\right), t \in[0,1]\right\}$.

Another critical case appears when the two species possess heterogeneous but proportional diffusion rates, mortality rate, and consumption rate, namely

Proposition 6.4 (Case of similar species). Suppose that $f_{1}=f_{2}$ and $A_{2}=\alpha A_{1}$ for some constant $\alpha \in \mathbb{R}_{+}^{*}$.

Then, for all $\left(c_{1}, c_{2}\right) \in \Theta$, we have the four relations

$$
R_{u}^{*}\left(c_{1}\right)=R_{v}^{*}\left(c_{2} / \alpha\right), \Theta=\emptyset, \widetilde{\Theta}=\left\{\left(c_{1}, \alpha c_{1}\right), c_{1} \geq c_{1}^{0}\right\}, c_{2}^{*}\left(c_{1}\right)=\alpha c_{1} .
$$


Moreover, the system has a coexistence solution $(R, U, V) \in\left(X_{+}^{*}\right)^{3}$ if and only if $\left(c_{1}, c_{2}\right) \in \widetilde{\Theta}$. In that case $\left\{\left(R_{u}^{*}, t U^{*},(1-t) U^{*}\right), t \in(0,1)\right\}$ is a family of solutions and each coexistence solution satisfies $(R, U, V) \in\left\{\left(R_{u}^{*}, t U^{*},(1-t) U^{*}\right), t \in(0,1)\right\}$.

Proof of Proposition 6.4. The system defining $\left(R_{u}^{*}\left(c_{1}\right), U^{*}\left(c_{1}\right)\right)$ is

$$
A_{1} U^{*}\left(c_{1}\right)-c_{1} f_{1}\left(R_{u}^{*}\left(c_{1}\right)\right) U^{*}\left(c_{1}\right)=0, \quad A_{0} R_{u}^{*}\left(c_{1}\right)+A_{1} U^{*}\left(c_{1}\right)=0,
$$

while the system defining $\left(R_{v}^{*}\left(c_{2}\right), V^{*}\left(c_{2}\right)\right)$ is in the present case

$$
\alpha A_{1} V^{*}\left(c_{2}\right)-c_{2} f_{1}\left(R_{v}^{*}\left(c_{2}\right)\right) V^{*}\left(c_{1}\right)=0, \quad A_{0} R_{v}^{*}\left(c_{2}\right)+\alpha A_{1} V^{*}\left(c_{2}\right)=0 .
$$

The uniqueness result of Proposition 4.4 provides $V^{*}\left(c_{2}\right)=\frac{1}{\alpha} U^{*}\left(\frac{c_{2}}{\alpha}\right)$, and $R_{v}^{*}\left(c_{2}\right)=$ $R_{u}^{*}\left(\frac{c_{2}}{\alpha}\right)$. Now, since $c_{2}^{*}\left(c_{1}\right)$ is defined as the unique value of the parameter $c_{2}$ such that $\lambda_{1}\left(A_{2}-c_{2} f_{2}\left(R_{u}^{*}\left(c_{1}\right)\right)\right)=0$, i.e. $\lambda_{1}\left(A_{1}-\left(c_{2} / \alpha\right) f_{1}\left(R_{u}^{*}\left(c_{1}\right)\right)\right)=0$, it comes $c_{2}^{*}\left(c_{1}\right)=\alpha c_{1}$. This together with the analogous relation for $c_{1}^{*}\left(c_{2}\right)$ provides

$$
\Theta=\emptyset, \quad \widetilde{\Theta}=\left\{\left(c_{1}, \alpha c_{1}\right) ; c_{1}>c_{1}^{0}\right\}
$$

Take now $\left(c_{1}, c_{2}\right)$ such that $(R, U, V)$ is an associated coexistence solution. We have

$$
\lambda_{1}\left(A_{1}-\frac{c_{2}}{\alpha} f_{1}(R)\right)=\lambda_{1}\left(A_{1}-c_{1} f_{1}(R)\right)=0,
$$

and monotone dependence of the above $\lambda_{1}$ 's with the parameters $c_{1}$ and $c_{2}$ implies $c_{2}=\alpha c_{1}$. Besides, summing the last two equations of 1.2 leads to

$$
\left\{\begin{array}{l}
A_{0} R+c_{1} f_{1}(R)(U+\alpha V)=I, \\
\left(A_{1}-c_{1} f_{1}(R)\right)(U+\alpha V)=0,
\end{array}\right.
$$

so that uniqueness provides $U+\alpha V=U^{*}\left(c_{1}\right)$, and $R=R_{u}^{*}\left(c_{1}\right)$. On top of that, coming back to the equations satisfied by $U$ resp. $V$, it appears that there exists $(t, y) \in \mathbb{R}_{+}^{2}$ such that $U=t U^{*}\left(c_{1}\right)$, and $\alpha V=y U^{*}\left(c_{1}\right)$. Gathering the relations then provides the necessary equation $t+y=1$. This ends the proof.

6.4 Coexistence domain when diffusion rates tend to $\infty$

For a small value of $\varepsilon>0$, we consider the system

$$
\left\{\begin{array}{l}
\left(m_{0}-\frac{a_{0}}{\varepsilon} \Delta\right) R+c_{1} f_{1}(R) U+c_{2} f_{2}(R) V=I \\
\left(m_{1}-\frac{a_{1}}{\varepsilon} \Delta\right) U-c_{1} f_{1}(R) U=0 \\
\left(m_{2}-\frac{a_{2}}{\varepsilon} \Delta\right) U-c_{2} f_{2}(R) V=0
\end{array}\right.
$$

with Neumann boundary condition ${ }^{27}$ It can be shown (see 25]), using the central manifold theorem, that the solution to 6.1 converges to the solution of the so-called aggregated system

$$
\left\{\begin{array}{l}
\widetilde{m_{0}} r+c_{1} \widetilde{f}_{1}(r) u+c_{2} \widetilde{f}_{2}(r) v=\widetilde{I} \\
\left(\widetilde{m_{1}}-c_{1} \widetilde{f}_{1}(r)\right) u=0 \\
\left(\widetilde{m_{2}}-c_{2} \widetilde{f}_{2}(r)\right) v=0
\end{array}\right.
$$

27 This is the only place in this text where Neumann - and not Robin - boundary conditions are required 
where $\widetilde{m_{i}}=\frac{1}{|\Omega|} \int_{\Omega} m_{i}(x) d x, \widetilde{f}_{i}(r)=\frac{1}{|\Omega|} \int_{\Omega} f_{i}(x, r), \widetilde{I}=\frac{1}{|\Omega|} \int_{\Omega} I(x) d x$, and the unknown $r, u, v$ now are scalars (independent of $x$ ). System 6.2 is a homogeneous chemostat system. Therefore, and as is easily seen on the equations, in the generic case there are no positive solution to $(6.2)$. As it is proved in [25, it turns out that for $\varepsilon>0$ small enough, the original system 6.1) has no positive solution in the generic case neither.

This result allows to describe the behavior of the coexistence domain $\Theta$ when the diffusion rates tend to $+\infty$. Remark in passing that, if Assumption 2 is true for a given $\varepsilon>0$, then it remains true for each $\varepsilon>0$. In this case, Theorem 2.16 shows that there exists $\Theta^{\varepsilon} \subset \mathbb{R}_{+}^{2}$ such that, for each $\left(c_{1}, c_{2}\right) \in \Theta^{\varepsilon}$, the system 6.1 admits a coexistence solution. The boundaries of $\widetilde{\Theta^{\varepsilon}}$ are given by the curves

$$
\left\{\left(c_{1}, c_{2}^{*, \varepsilon}\left(c_{1}\right)\right) ; c_{1}>c_{1}^{0, \varepsilon}\right\} \quad \text { and } \quad\left\{\left(c_{1}^{*, \varepsilon}\left(c_{2}\right), c_{2} ; c_{2}>c_{2}^{0, \varepsilon}\right\} .\right.
$$

Define for convenience the quantity $r_{i}^{*}\left(c_{i}\right)$ as

$$
r_{i}^{*}\left(c_{i}\right)=\tilde{f}_{i}^{-1}\left(m_{i} / c_{i}\right) \text { if this is well-defined, and } r_{i}^{*}\left(c_{i}\right)=+\infty \text { otherwise. }
$$

We have the

Proposition 6.5 Denote $\Theta^{\infty}=\left\{\left(c_{1}, c_{2}\right)\right.$ s.t. $\left.r_{1}^{*}\left(c_{1}\right)=r_{2}^{*}\left(c_{2}\right)<+\infty\right\}$.

Then, for each $\left(c_{1}, c_{2}\right) \notin \Theta^{\infty}$, there exists $\varepsilon_{0}>0$ such that $\forall \varepsilon \in\left(0, \varepsilon_{0}\right)$, we have $\left(c_{1}, c_{2}\right) \notin \Theta^{\varepsilon}$.

Proof of Proposition 6.5. If $\left(c_{1}, c_{2}\right) \notin \Theta^{\infty}$, then $r_{1}^{*}\left(c_{1}\right) \neq r_{2}^{*}\left(c_{2}\right)$. Therefore, the system 6.2 has no positive solution and this implies (25]) that there exists $\varepsilon_{0}>0$ such that, for all $\varepsilon \in\left(0, \varepsilon_{0}\right)$, the system 6.2 has no positive solution. Hence, $\left(c_{1}, c_{2}\right) \notin \Theta^{\varepsilon}$ if $\varepsilon$ is small enough.

In this sense, the coexistence domain $\Theta^{\varepsilon}$ tends to the curve $\Theta^{\infty}$ when $\varepsilon \rightarrow 0$. As the diffusion rates increase, the aggregation phenomena leads to system that is close to homogeneous in space, and the coexistence domain shrinks to a curve.

\section{Conclusion and perspectives}

This study examines a model where two species compete for a single resource, in a spatially heterogeneous domain. Our system differs from the classical unstirred chemostat system [13, 12, 11, 3, 18, in that the the reaction terms do depend on space, and, more importantly, we allow the diffusion rates to depend on the species under consideration. This point leads to a new mathematical difficulty. Namely, the conservation law which links the resource $R$ with the two species $U$ and $V$, noted $A_{0} R+A_{1} U+A_{2} V=I$ in the core of the paper, becomes a nonlocal equation (as compared to the previously quoted papers where the analogous equation is local). We circumvent this difficulty by introducing Assumption 2 (supplemented with Assumption 1.6 in the case of Robin boundary conditions).

We show that coexistence occurs when the consumption parameters $\left(c_{1}, c_{2}\right)$ lie in a subdomain $\Theta \subset \mathbb{R}_{+}^{2}$. In addition, we study the set $\Theta$ by using a characterisation of $\Theta$ that relies on the two functions $c_{1}^{*}\left(c_{2}\right)$ and $c_{2}^{*}\left(c_{1}\right)$ defined in the text.

Several direction may extend this study. Firstly, our numerical observations indicate that the coexistence solution are non-degenerate, except in the particular case when the 
two functions $c_{1}^{*}($.$) and c_{2}^{*}($.$) coincide. When the coexistence solution is non-degenerate,$ it turns out that our construction can be extended to three species, and by iteration, to $N$ species for any value of $N$. It would therefore be a key step to actually prove that the coexistence solutions necessarily are non-degenerate, unless $c_{1}^{*}($.$) and c_{2}^{*}($.$) coincide$ Note in passing that Propositions 6.3 and 6.4 give two examples of situations where $c_{1}^{*}($.$) and c_{2}^{*}($.$) coincide, and a complete description of the coexistence phenomena is$ provided in these situations.

Secondly, we defined $\Theta$ as the union of two subdomain $\Theta_{-}$and $\Theta_{+}$. If $\left(c_{1}, c_{2}\right) \in \Theta_{-}$ then $c_{i}^{*}>c_{i}^{* *}$ and the bifurcation occurs "to the left" (see Figure 2.2. . We conjecture that $\Theta_{-}=\emptyset$ in any case. In fact, to rephrase our conjecture, if $\left(c_{1}, c_{2}\right) \in \Theta_{-}$, then both species are "not invasive" in the sense that

$$
\lambda_{1}\left(A _ { 1 } - c _ { 1 } f _ { 1 } ( R _ { v } ^ { * } ( c _ { 2 } ) ) < 0 \text { , and } \lambda _ { 1 } \left(A_{2}-c_{2} f_{2}\left(R_{u}^{*}\left(c_{1}\right)\right)<0 .\right.\right.
$$

Note that Waltmann et al. 11] formulate a similar conjecture. Namely, they conjecture that a necessary condition for two species to coexist is that both species are "invasive" in the sense that $\lambda_{1}\left(A_{1}-c_{1} f_{1}\left(R_{v}^{*}\left(c_{2}\right)\right) \geq 0\right.$ and $\lambda_{1}\left(A_{2}-c_{2} f_{2}\left(R_{u}^{*}\left(c_{1}\right)\right) \geq 0\right.$. Lastly, note that if $\left(c_{1}, c_{2}\right) \in \Theta_{-}$, then the index of both semi-trivial solutions is equal to 1. To rephrase the above considerations, Waltmann et al. in [11] conjecture that a necessary condition for two species to coexist is that both sem-trivial solution are unstable (for the time-dependent problem), which in our case, means that the index of the two semi-trivial solutions is equal to -1 . Note that even if the latter result is proved, it is not clear that the coexistence solution itself is stable. Indeed, Hofbauer and So 17. show that there exists gradostats (that is, similar models with a discrete spatial structuration) for which an unstable coexistence solution may be exhibited. A more precise description of $\Theta$ would be a first step to understand the situation.

Thirdly, we conjecture that if $\left(c_{1}, c_{2}\right) \notin \widetilde{\Theta}$, then no coexistence solution can be found. Would this result be proved, we could use $\Theta$ as a geometrical indicator of the possibility of coexistence in a given system. Numerical investigations on the relation between $\Theta$, spatial heterogeneity, and the biodiversity, will be published soon.

Finally, our proof uses basically Assumption 2, an assumption that allows us to extend the analysis of the (known) case where all diffusion operators coincide. It is to be noted, however, that our approach proves the existence of semi-trivial solutions without using Assumption 2 This assumption is only needed to obtain uniqueness and non-degeneracy of the so-obtained semi-trivial solutions. A natural question is: can one extend our construction to situations where Assumption 2 is not verified?

\section{References}

1. M. G. Crandall and P. H. Rabinowitz, Bifurcation from simple eigenvalue, J. Func. Anal., 8 (1971), 321-340.

2. P. H. Rabinowitz, Some global results for nonlinear eigenvalue problems, J. Func. Anal., 7 (1971), 487-513.

3. H. L. Smith and P. Waltman, The theory of the Chemostat, Cambridge university press, 1995.

4. A. Ambrosetti and A. Malchiodi, Nonlinear Analysis and Semilinear elliptic problems, Cambrige university press, 2007.

5. J. Smoller, Shock Waves and Reaction-diffusion Equations, Springer-Verlag, 1993.

6. C.V. Pao, Quasisolutions and global attractor of reaction-diffusion systems, Nonlinear Ana., Theory, Methods and Applications, 26, 1996, 1889-1903. 
7. C.V. Pao, On nonlinear reaction-diffusion equations, J. Math. Analysis applic, 87, 1982, $165-198$.

8. C. Walker, Coexistence Steady States in a Predator-Prey Model, Arch. Math., 95, 2010, 87-99.

9. C. Walker, Global Bifurcation of Positive Equilibria in Nonlinear Population Models, J. Differential Equations, 248, 2010, 1756-1776.

10. Z. Zhang, Coexistence and Stability of Solutions for a Class of Reaction-Diffusion Systems, E. J . Diff. Eq., 137, 2005, 1-16.

11. S. B. Hsu and P. Waltman, On a system of reaction-diffusion equations arising from competition in an unstirred chemostat., SIAM J. Appl. Math, 53, 1993, 1026-1044.

12. S.B. Hsu, H. Smith and P. Waltman, Dynamic of competition in the unstirred chemostat., Can. Appl. Math. Quart., 2, 1994, 461-483.

13. J. L. Dung, H. L. Smith and P. Waltman, Growth in the unstirred chemostat with different diffusion rates, Fields institute communications, 21, 1999, 131-142.

14. S. Zheng and J. Liu, Coexistence solutions for a reaction-diffusion system of un-sirred chemostat model, Applied Math. and Comp., 145, 2003, 579-590.

15. S. Zheng and H. Guo and J. Liu, A food chain model for two resources in unstirred chemostat, Applied Math. Comp., 206, 2008, 389-402.

16. J. V. Baxley and S. B. Robinson, Coexistence in the unstirred chemosat, Applied Math. And Comput., 39, 1998, 41-65.

17. J. Hofbauer and J. W. H. So, Competition in the gradostat: The global stability problem, Nonlinear analysis, 22, 1994, 1017-1033.

18. J. H. Wu, Global bifurcation of coexistence state for the competition model in the chemostat, Nonlinear Analysis, 39, 2000, 817-835.

19. H. Nie and J. Wu: Uniqueness and stability for coexistence solutions of the unstirred chemostat model, Applicable Analysis 89, 1151-1159 (2010)

20. J. Blat and K. J. Brown, Bifurcation of steady-state solutions in predator-prey and competition systems, Proc. Roy. Soc. Edin.A, 97, 1984, 21-34.

21. J. Blat and K. J. Brown, Global bifurcation of positive solutions in some systems of elliptic equations, SIAM J. Math. Anal., 17, 1986, 1339-1353.

22. Y. Du end K. J. Brown,Bifurcation and Monotonicity in Competition Reaction-Diffusion Systems, Nonlinear Ana. Th. Meth. \& Appl., 23 No. 1, 1994, 1-13.

23. E. D. Conway, Diffusion and The Predator-Prey Interaction: Steady States with Flux at the Boundaries, Contemporary. Math., 17, 1983, 215-234.

24. E. Dancer, On positive solutions of some partial differential equations, Trans. Amer. Math. Soc., 284, 1984

25. S. Madec and F. Castella, Global behavior of $N$ competiting species with strong diffusion: diffusion leads to exclusion, to appear 